\title{
The diverse habitats of Hygrocybe - peeking into an enigmatic lifestyle
}

\author{
Halbwachs $\mathbf{H}^{1 *}$, Karasch $\mathrm{P}^{2}$ and Griffith $\mathrm{GW}^{3}$ \\ ${ }^{I}$ Bavarian Mycological Society, Danzigerstr. 20, D-63916 Amorbach, Germany \\ ${ }^{2}$ Bavarian Mycological Society, Taubenhüller Weg 2A, D - 82131 Gauting, Germany \\ ${ }^{3}$ Institute of Biological, Environmental and Rural Sciences, Cledwyn Building, Aberystwyth University, Penglais, \\ Aberystwyth, Ceredigion SY23 3DD Wales, gwg@aber.ac.uk
}

Halbwachs H, Karasch P, Griffith GW 2013 - The diverse habitats of Hygrocybe - peeking into an enigmatic lifestyle. Mycosphere 4(4), 773-792, Doi 10.5943/mycosphere/4/4/14

\begin{abstract}
The genus Hygrocybe (Fr.) P. Kumm. is globally distributed. Certain taxa occur on different continents, even in the northern and the southern hemisphere. In Europe grassland is the preferred habitat. The trophic ecology is still unclear. In this review studies and internet sources of about 65 locations distributed from high to low latitudes across all continents in which 16 Hygrocybe species (typically found in Europe) occur have been analysed with regard to habitat types, vegetation and soil properties. The analysis shows that several of the selected Hygrocybe species can cope with a very wide range of habitats. They appear to prefer soils with low fertility, grassland over forests and temperate/boreal climates. Most significantly, waxcaps are found in forest habitats in the absence of grasses, and in grasslands where woody hosts are absent. Mosses are not obligately associated. Their host associations are extremely flexible. A biotrophic lifestyle should be considered.
\end{abstract}

Keywords - biotrophy - distribution - ecozones - grassland - mosses - soil - trophic ecology waxcaps - woodland

\section{Introduction}

The genus Hygrocybe (Fr.) P. Kumm. has a worldwide distribution (Beisenherz 2000, Krieglsteiner \& Gminder 2001, Boertmann 2010), except for arid ecozones (Beisenherz 2000). Hygrocybe (waxcaps) are found in forests, grassland, marshes, fens and bogs (Boertmann 2010). Typically, European waxcaps occur on extensively managed grassland and meadows (Boertmann 2010).

It has been argued that European grassland environments may be ersatz habitats, and that the primary habitats would be forests (Homola et al. 1985, Krieglsteiner 2004.). To our knowledge, conclusive evidence for this assumption has not been scientifically documented. In contrast, Vera (2000) proposed that during the early post-glacial, large herbivores (e.g. deer, bison, aurochs and wild horses) had been important in maintaining an open landscape including grassland (see also Griffith et al. 2004).

The nutritional mode of waxcaps is uncertain (Bresinsky 2008: 37f). To date they are mostly classified as saprotrophic (e.g. Knudsen \& Vesterholt 2012: 265), and more specifically being dependent on humic compounds in the soil (Arnolds 1981). However, as noted by Seitzman et al. (2011), many related taxa within the family Hygrophoraceae exhibit biotrophic nutritional 
strategies, including ectomycorrhizas (Hygrophorus) and lichenised taxa (Lichenomphalia) which is supported by the stable isotope signatures of waxcaps (Griffith et al. 2002, Seitzman et al. 2011). The latter authors have also suggested that waxcaps might be associated with bryophytes as hosts. All waxcaps share one clear-cut property - they cannot be grown under axenic laboratory conditions (Griffith et al. 2002, Halbwachs unpublished data), which is a hallmark of most biotrophic fungi, e.g. obligate parasites (Cooke 1979: 39).

Trophic modes are to a certain extent related to habitats. Ectomycorrhizal fungi for example, are untypical for grasslands, because they only rarely harbour specific host plants (cf. Eriksen et al. 2002). The spectrum of habitats "European" waxcaps inhabit has not been analysed to date in a systematic manner. To shed more light onto the question, whether the range of habitats can tell us something about the nutritional lifestyle of (European) waxcaps, we analysed in this review 65 studies and internet-based sources about sites in Europe, the Americas, Africa, Macaronesia, Asia, the Arctic and Antarctic, Australia and New Zealand.

\section{Methods}

To compare habitats, we focused on Hygrocybe spp. sensu Boertmann (2010). These included the ten most common species and species complexes in Europe: H. ceracea, $H$. chlorophana, $H$. coccinea s.l., $H$. conica s.l., $H$. insipida, H. pratensis s.l., $H$. psittacina s.l., $H$. punicea, $H$. quieta and $H$. virginea s.l., based on the total number of records for each species on the Field Records Database of Britain and Ireland (BMS 2012) and which correlated well with occurrence in Germany (Bresinsky 2008), as well as six less common species, which nevertheless are widely distributed ( $H$. aurantiosplendens, $H$. flavipes, $H$. fornicata, $H$. miniata s.l., $H$. mucronella, $H$. nitrata).

We attempted to compare as many diverse habitats as possible where common European Hygrocybe taxa occur. This turned out to be amazingly difficult, because important habitat information about climatic characteristics, associated vegetation and soil properties are either rarely recorded, or not recorded in a sytematic and consistent manner. Nevertheless, we were able to compare 65 waxcap sites worldwide (Fig. 1) by using additional sources, such as data banks and internet-based site descriptions providing data on vegetation and soil. To compare the climatic influence we assigned the sites to ecozones as defined by FAO (2001). We included data from two German sites and from a site on La Palma (Canaries), which we had compiled personally in 2011 and 2005 (unpublished data).

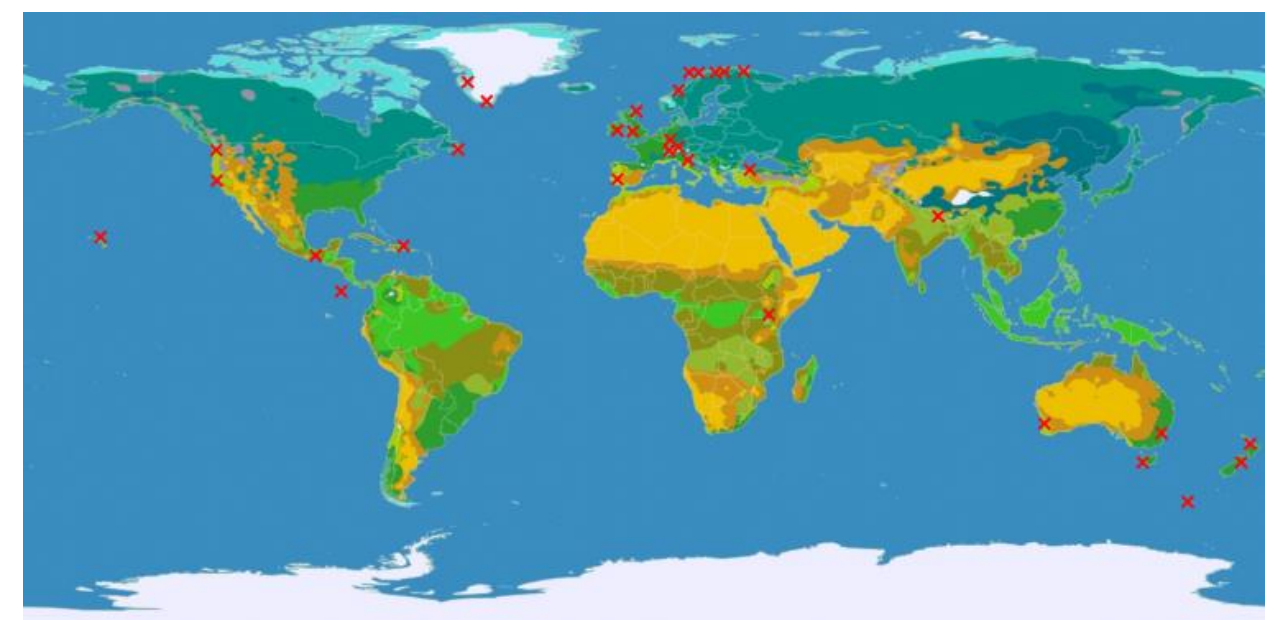

Fig. 1 - Map with simplified eco-zones according to Köppen (1931), showing the global distribution of sites for which Hygrocybe spp. data were obtained. Red X indicate the waxcap site areas covered by the study. Map source: Wikimedia Commons 2007; author LordToran. Legend: tropical rainforest, savannah, steppe, desert, humid temperate, humid subtropical, humid continental, transsiberian, dry-cold summer, tundra, glaciated-polar. 
There are clearly more data for Europe than for other regions, in large part due to higher human density and long history of mycological study, so care was taken to select sites across all ecozones of the continent. In this way a possible selection bias was minimised. The data we compiled comprised ecozones, habitat descriptions, associated vegetation and soil properties (see master table in table 1).

Climate zones were recorded according to the ecozone concept, as it has been developed by various authors (e.g. Köppen 1931, Walter \& Breckle 1999, Schultz 2008). We chose the climatecentred modified Köppen-Trewartha scheme, which is used by FAO (2001). This has been found to exhibit a good correspondence between its sub-zones or climatic types and the natural climax vegetation types and soils within them (Bailey 1996; Kehl 2011). This approach allows a standardised evaluation of the climatic bandwidth, in which the selected waxcap species occur. If not already stated in the source data, elevation and geographical coordinates were determined with Google Maps.

Vegetation, if not sufficiently mentioned in the source, was determined by internet-based national vegetation charts or in some cases site descriptions where waxcap sites were located within protected areas. Of particular interest were data about woodland and grassland types characterising the various habitats.

For additional information about vegetation preferences of waxcaps we analysed 220 internet images from 17 countries in Asia, Australasia, Europe, North and Central America. Typical images are shown in Figure 2.

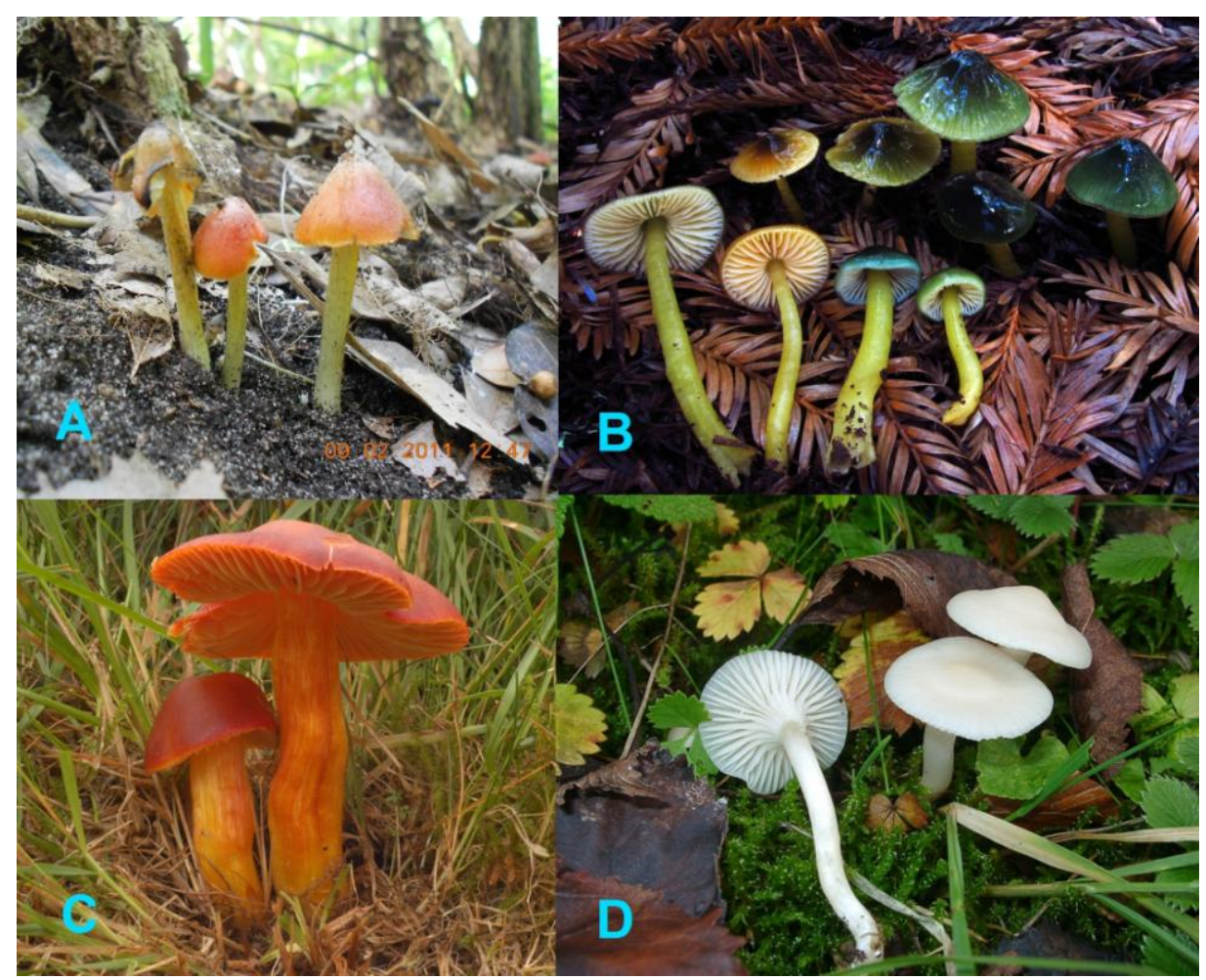

Fig. 2 - Examples of internet images used for determining the main plant groups associated with waxcaps. A: H. conica (Florida USA, () Richard Kneal - common domain); B: H. psittacina (California USA, (C) Erin Page Blanchard - common domain); C: H. punicea (Derbyshire UK, (C) Malcolm Storey - courtesy of M. Storey); D: H. virginea (Black Forest Germany, (C Georg Müller - courtesy of G. Müller).

Image sources: http://mushroomobserver.org/observer/observation_search?pattern=hygrocybe, http://www.bioimages. org.uk/, http://www.wildaboutbritain.co.uk/fungi, http://www.in2.dk/svampe/svpic/frame1.htm, http://www.mycokey. com/, http://www.pilzepilze.de/, http://www.pilzbestimmung.de/ 
Table 1 - Master table showing the investigated sites (column 2), their corresponding ecozones according to FAO (2001) (Column 1), habitat descriptions (column 3), predominant vegetation (column 4), soil types according to IUSS (2006) (column 5) and the primary sources (column 6).

\begin{tabular}{|c|c|c|c|c|c|}
\hline Ecozone FAO & Site & Habitat & Vegetation & Soil & Sources* \\
\hline $\begin{array}{l}\text { Boreal Coniferous Forests } \\
\text { (Ba): Boreal Plains }\end{array}$ & $\begin{array}{l}\text { Canada } \\
\text { Labrador, Avalon Peninsula, } \\
\text { Cape St. Mary's Park Reserve } \\
(46.83,-54.18) 100 \mathrm{~m}\end{array}$ & $\begin{array}{l}\text { Maritime Barrens Ecoregion } \\
\text { (Southeastern) }\end{array}$ & $\begin{array}{l}\text { heathlands, yellow birch (Betula } \\
\text { alleghaniensis) }\end{array}$ & $\begin{array}{l}\text { orthic podzols, dystric gleysols, } \\
\text { dystric histosols, lithosols }(50 \text {, } \\
30,10,10)\end{array}$ & $\begin{array}{l}\text { DFRA 2002, } \\
\text { Voitk } 2005\end{array}$ \\
\hline $\begin{array}{l}\text { Boreal Coniferous Forests } \\
\text { (Ba): Boreal Plains }\end{array}$ & $\begin{array}{l}\text { Canada } \\
\text { Labrador, Avalon Peninsula, La } \\
\text { Manche Provincial Park (47.18, } \\
\text {-52.89) } 80 \mathrm{~m}\end{array}$ & $\begin{array}{l}\text { Maritime Barrens Ecoregion } \\
\text { (Southeastern), Avalon } \\
\text { Forest Ecoregion }\end{array}$ & $\begin{array}{l}\text { balsam fir (A. balsamea), yellow } \\
\text { birch (B. alleghaniensis) }\end{array}$ & $\begin{array}{l}\text { orthic podzols, dystric gleysols, } \\
\text { dystric histosols, lithosols }(50 \text {, } \\
30,10,10)\end{array}$ & $\begin{array}{l}\text { DFRA 2002, } \\
\text { Voitk } 2005\end{array}$ \\
\hline $\begin{array}{l}\text { Boreal Mountain Systems } \\
\text { (BM): Atlantic dwarf } \\
\text { shrub heaths }\end{array}$ & $\begin{array}{l}\text { Norway } \\
\text { Andøy, Høyvik }(69.24,15.87) \\
100 \mathrm{~m}\end{array}$ & natural grassland near beach & Poaceae & $\begin{array}{l}\text { dystric histosol, cambic } \\
\text { arenosol, lithosol }(70,20,10)\end{array}$ & NBIC 2011 \\
\hline $\begin{array}{l}\text { Boreal Mountain Systems } \\
\text { (BM): Atlantic dwarf } \\
\text { shrub heaths }\end{array}$ & $\begin{array}{l}\text { Norway } \\
\text { Andøy, Kobbedalen, } \\
\emptyset \text { verdalsplassen }(69.02,15.6) \\
11 \mathrm{~m}\end{array}$ & natural grassland & Poaceae & $\begin{array}{l}\text { dystric histosol, cambic } \\
\text { arenosol, lithosol }(70,20,10)\end{array}$ & NBIC 2011 \\
\hline $\begin{array}{l}\text { Boreal Mountain Systems } \\
\text { (BM): Atlantic dwarf } \\
\text { shrub heaths }\end{array}$ & $\begin{array}{l}\text { Norway } \\
\text { Nordland, Alstahaug, Tjøtta } \\
(65.83,12.43) 5 \mathrm{~m}\end{array}$ & $\begin{array}{l}\text { meadows, small birch, salt } \\
\text { marsh }\end{array}$ & Poaceae, herbs & $\begin{array}{l}\text { lithosol, dystric regosol, } \\
\text { cambric arenosol }(60,20,20)\end{array}$ & NBIC 2011 \\
\hline $\begin{array}{l}\text { Boreal Mountain Systems } \\
\text { (BM): Atlantic dwarf } \\
\text { shrub heaths }\end{array}$ & $\begin{array}{l}\text { Norway } \\
\text { Nordland, Dønna, Sandstrak } \\
(66.06,12.39) 0-40 \mathrm{~m}\end{array}$ & $\begin{array}{l}\text { meadow in saltmarsh, } \\
\text { heathland, birch forest }\end{array}$ & birch, Dryas, juniper & $\begin{array}{l}\text { lithosol, dystric regosol, } \\
\text { cambric arenosol }(40,40,20)\end{array}$ & NBIC 2011 \\
\hline $\begin{array}{l}\text { Boreal Mountain Systems } \\
\text { (BM): Atlantic dwarf } \\
\text { shrub heaths }\end{array}$ & $\begin{array}{l}\text { Norway } \\
\text { Nordland, Evenes, Evenesmarka } \\
(68.50,16.76) 80 \mathrm{~m}\end{array}$ & $\begin{array}{l}\text { natural grassland on } \\
\text { calcareous soils }\end{array}$ & Poaceae & $\begin{array}{l}\text { eutric cambisols, lithic } \\
\text { leptosols, haplic podzols, } \\
\text { dystric fluvisols, umbric } \\
\text { gleysols }(40,20,20,10,10)\end{array}$ & NBIC 2011 \\
\hline $\begin{array}{l}\text { Boreal Mountain Systems } \\
\text { (BM): Atlantic dwarf } \\
\text { shrub heaths }\end{array}$ & $\begin{array}{l}\text { Norway } \\
\text { Troms, Lyngen, Lyngsdalen, } \\
\text { Trange }(69.44,20.12) 130 \mathrm{~m}\end{array}$ & stony grassland & Poaceae & $\begin{array}{l}\text { haplic podzols, dystric regosols, } \\
\text { lithic leptosols, gleyic podzols } \\
(40,30,20,10)\end{array}$ & NBIC 2011 \\
\hline $\begin{array}{l}\text { Boreal Mountain Systems } \\
\text { (BM): Atlantic dwarf } \\
\text { shrub heaths }\end{array}$ & $\begin{array}{l}\text { Norway } \\
\text { Troms, west of Harstad (68.80, } \\
16.45) 160-200 \mathrm{~m}\end{array}$ & grassy slope & Poaceae & $\begin{array}{l}\text { orthic podzols, dystric regosol, } \\
\text { lithosol, gleyic podzol }(40,30 \text {, } \\
20,10)\end{array}$ & NBIC 2011 \\
\hline $\begin{array}{l}\text { Boreal Mountain Systems } \\
\text { (BM): Western boreal and } \\
\text { montane birch forests, } \\
\text { with pine }\end{array}$ & $\begin{array}{l}\text { Norway } \\
\text { Finnmark, Alta, Vassbotndalen } \\
\text { v. Bjørnelva }(69.99,22.85) 75 \\
\text { m }\end{array}$ & grassy trail & Poaceae & $\begin{array}{l}\text { dystric regosol, orthic podzols, } \\
\text { lithosol }(40,40,20)\end{array}$ & NBIC 2011 \\
\hline
\end{tabular}




\begin{tabular}{|c|c|c|c|c|c|}
\hline Ecozone FAO & Site & Habitat & Vegetation & Soil & Sources* \\
\hline $\begin{array}{l}\text { Boreal Mountain Systems } \\
(\mathrm{BM}) \text { : Western boreal and } \\
\text { montane birch forests, } \\
\text { with pine }\end{array}$ & $\begin{array}{l}\text { Norway } \\
\text { Finnmark, Sør-Varanger, } \\
\text { Melkefoss (upstream river } \\
\text { Pasvik, plot gg4) (69.40, 29.76) } \\
40 \mathrm{~m}\end{array}$ & former pasture on sandy soils & Poaceae & $\begin{array}{l}\text { orthic podzols, dystric regosol, } \\
\text { dystric histosol, gleyic podzol } \\
(50,20,20,10)\end{array}$ & NBIC 2011 \\
\hline $\begin{array}{l}\text { Boreal Mountain Systems } \\
(\mathrm{BM}) \text { : Western boreal and } \\
\text { montane birch forests, } \\
\text { with pine }\end{array}$ & $\begin{array}{l}\text { Norway } \\
\text { Finnmark, Sør-Varanger, } \\
\text { Noatun, courtyard }(69.16,29.25) \\
50 \mathrm{~m}\end{array}$ & grassland with birch and pine & Poaceae, Betula, Pinus & $\begin{array}{l}\text { orthic podzols, dystric regosol, } \\
\text { dystric histosol, gleyic podzol } \\
(50,20,20,10) \text {, glacial river } \\
\text { deposits }\end{array}$ & NBIC 2011 \\
\hline $\begin{array}{l}\text { Boreal Mountain Systems } \\
\text { (BM): Western boreal and } \\
\text { montane birch forests, } \\
\text { with pine }\end{array}$ & $\begin{array}{l}\text { Norway } \\
\text { Nordland, Evenes, Botn }(68.49 \text {, } \\
17.11) 7 \mathrm{~m}\end{array}$ & grassland with trees & $\begin{array}{l}\text { birch, alder, aspen, pine, willow, } \\
\text { Poaceae }\end{array}$ & $\begin{array}{l}\text { haplic podzols, dystric regosols, } \\
\text { lithic leptosols, gleyic podzols } \\
(40,30,20,10)\end{array}$ & NBIC 2011 \\
\hline $\begin{array}{l}\text { Boreal Mountain Systems } \\
(\mathrm{BM}) \text { : Western boreal and } \\
\text { montane birch forests, } \\
\text { with pine }\end{array}$ & $\begin{array}{l}\text { Norway } \\
\text { Nordland, Sortland: } \\
\text { Holmstaddalen }(68.71,15.31) \\
160 \mathrm{~m}\end{array}$ & $\begin{array}{l}\text { natural meadow (forest } \\
\text { meadow) }\end{array}$ & $\begin{array}{l}\text { birch, alder, aspen, pine, willow, } \\
\text { Poaceae }\end{array}$ & $\begin{array}{l}\text { lithosols, dystric regosols, } \\
\text { cambic arenosols }(60,20,20)\end{array}$ & NBIC 2011 \\
\hline $\begin{array}{l}\text { Boreal Mountain Systems } \\
(\mathrm{BM}) \text { : Western boreal and } \\
\text { montane birch forests, } \\
\text { with pine }\end{array}$ & $\begin{array}{l}\text { Norway } \\
\text { Troms, Bardu, Øverland }(68.70 \text {, } \\
\text { 18.10) } 250 \mathrm{~m}\end{array}$ & $\begin{array}{l}\text { between herbs on gravelroad } \\
\text { shoulder }\end{array}$ & $\begin{array}{l}\text { herbs, downy birch (Betula } \\
\text { pubescens subsp. tortuosa) }\end{array}$ & $\begin{array}{l}\text { dystric regosol, orthic podzols, } \\
\text { lithosol }(40,40,20)\end{array}$ & $\begin{array}{l}\text { NBIC 2011, } \\
\text { Stabursvik } \\
2007\end{array}$ \\
\hline $\begin{array}{l}\text { Boreal Mountain Systems } \\
\text { (BM): Western boreal and } \\
\text { montane birch forests, } \\
\text { with pine }\end{array}$ & $\begin{array}{l}\text { Norway } \\
\text { Troms, Kåfjord, Kåfjorddalen, } \\
\text { Ankerlia }(69.43,20.97) \text { 120-180 } \\
\text { m }\end{array}$ & subalpine birch forest & Betula & $\begin{array}{l}\text { dystric regosols, haplic podzols, } \\
\text { lithic leptosols }(40,40,20)\end{array}$ & NBIC 2011 \\
\hline $\begin{array}{l}\text { Boreal Mountain Systems } \\
\text { (BM): Western boreal and } \\
\text { montane birch forests, } \\
\text { with pine }\end{array}$ & $\begin{array}{l}\text { Norway } \\
\text { Troms, Nordreisa, Sappen, near } \\
\text { school }(69.56,21.29) 74 \mathrm{~m}\end{array}$ & pine forest & Pinus & $\begin{array}{l}\text { haplic podzols, dystric regosols, } \\
\text { fibric histosols, gleyic podzols } \\
(50,20,20,10)\end{array}$ & NBIC 2011 \\
\hline $\begin{array}{l}\text { Boreal Mountain Systems } \\
\text { (BM): Western boreal and } \\
\text { montane birch forests, } \\
\text { with pine }\end{array}$ & $\begin{array}{l}\text { Norway } \\
\text { Troms, Storfjord, Skibotn, near } \\
\text { Kurssenter }(69.39,20.27) 1-10 \\
\text { m }\end{array}$ & oligotrophic pine forest & pine & $\begin{array}{l}\text { dystric fluvisol, cambic } \\
\text { arenosol }(60,40)\end{array}$ & NBIC 2011 \\
\hline
\end{tabular}




\begin{tabular}{|c|c|c|c|c|c|}
\hline Ecozone FAO & Site & Habitat & Vegetation & Soil & Sources* \\
\hline $\begin{array}{l}\text { Boreal Tundra Woodland } \\
\text { (Bb) }\end{array}$ & $\begin{array}{l}\text { Canada } \\
\text { Labrador, Avalon Peninsula, } \\
\text { Salmonier Nature Park (47.26, - } \\
\text { 53.28) } 130 \mathrm{~m}\end{array}$ & $\begin{array}{l}\text { Maritime Barrens Ecoregion } \\
\text { (Southeastern), Avalon } \\
\text { Forest Ecoregion }\end{array}$ & $\begin{array}{l}\text { Abies balsamea, Betula } \\
\text { alleghaniensis, heathlands }\end{array}$ & $\begin{array}{l}\text { orthic podzols, dystric gleysols, } \\
\text { dystric histosols, lithosols }(50, \\
30,10,10)\end{array}$ & $\begin{array}{l}\text { DFRA 2002, } \\
\text { Voitk } 2005\end{array}$ \\
\hline $\begin{array}{l}\text { Cascade Mixed Forest- } \\
\text { Coniferous Forest-Alpine } \\
\text { Meadow (TeM / M242) }\end{array}$ & $\begin{array}{l}\text { USA } \\
\text { Cooper Point Rd. NW and 68th } \\
\text { Ave. NW, Olympia, Washington } \\
(47.13,-122.93) 50 \mathrm{~m}\end{array}$ & $\begin{array}{l}\text { in abundance under Western } \\
\text { red cedar }\end{array}$ & Thuja plicata & $\begin{array}{l}\text { humic acrisols, haplic } \\
\text { phaeozems, humic cambisols } \\
(60,20,20)\end{array}$ & $\begin{array}{l}\text { TOA 2011a, } \\
\text { Bailey } 1995\end{array}$ \\
\hline $\begin{array}{l}\text { Polar Arctic Tundras (P), } \\
\text { Boreal Subarctic Tundras } \\
(\mathrm{Ba})\end{array}$ & $\begin{array}{l}\text { Greenland } \\
\text { Fredericshab, head of Eqaluit } \\
\text { fjord }(61.99,-49.53) 20 \mathrm{~m}\end{array}$ & herb and grassland slopes & $\begin{array}{l}\text { Alchemilla, Setaria glauca, } \\
\text { Juniperus }\end{array}$ & Gelic regosols & $\begin{array}{l}\text { Borgen \& } \\
\text { Arnolds } \\
2004\end{array}$ \\
\hline $\begin{array}{l}\text { Polar Arctic Tundras (P), } \\
\text { Boreal Subarctic Tundras } \\
(\mathrm{Ba})\end{array}$ & $\begin{array}{l}\text { Greenland } \\
\text { Igaliku }(60.98,-45.43) 40 \mathrm{~m}\end{array}$ & $\begin{array}{l}\text { grassland slope, grazed by } \\
\text { sheep }\end{array}$ & $\begin{array}{l}\text { Poaceae, Betula glandulosa, Setaria } \\
\text { glauca }\end{array}$ & Gelic regosols & $\begin{array}{l}\text { Borgen \& } \\
\text { Arnolds } \\
2004\end{array}$ \\
\hline $\begin{array}{l}\text { Polar Arctic Tundras }(\mathrm{P}) \text {, } \\
\text { Boreal Subarctic Tundras } \\
(\mathrm{Ba})\end{array}$ & $\begin{array}{l}\text { Greenland } \\
\text { Nasarsuaq, Kiattuut Qaqqaat, } \\
\text { North of main river }(61.21,- \\
45.37) 150 \mathrm{~m}\end{array}$ & $\begin{array}{l}\text { rather dry, semi-natural } \\
\text { grassland slopes }\end{array}$ & Poaceae & Gelic regosols & $\begin{array}{l}\text { Borgen \& } \\
\text { Arnolds } \\
2004\end{array}$ \\
\hline $\begin{array}{l}\text { Polar Arctic Tundras }(\mathrm{P}), \\
\text { Boreal Subarctic Tundras } \\
(\mathrm{Ba})\end{array}$ & $\begin{array}{l}\text { Greenland } \\
\text { Nasarsuaq, near harbour }(61.15 \text {, } \\
-45.43) 10-60 \mathrm{~m}\end{array}$ & $\begin{array}{l}\text { among grasses, lichens and } \\
\text { mosses, dwarf-shrub copse }\end{array}$ & Thalictrum, Cyperaceae & Gelic regosols & $\begin{array}{l}\text { Borgen \& } \\
\text { Arnolds } \\
2004\end{array}$ \\
\hline $\begin{array}{l}\text { Polar Arctic Tundras }(\mathrm{P}) \text {, } \\
\text { Boreal Subarctic Tundras } \\
(\mathrm{Ba})\end{array}$ & $\begin{array}{l}\text { Greenland } \\
\text { Nuuk (Godthåb), Qooqqut } \\
\text { (Qôrqut) }(64.27,-50.90) 50 \mathrm{~m}\end{array}$ & $\begin{array}{l}\text { acidic, seminatural grassland } \\
\text { slope, on mesic to dry soil on } \\
\text { old river banks }\end{array}$ & $\begin{array}{l}\text { Carex, Empetrum, Salix herbacea, } \\
\text { Bryophyta }\end{array}$ & Gelic regosols & $\begin{array}{l}\text { Borgen \& } \\
\text { Arnolds } \\
2004\end{array}$ \\
\hline $\begin{array}{l}\text { Polar, Antarctic Tundras } \\
\text { (P) }\end{array}$ & $\begin{array}{l}\text { Antarctica } \\
\text { Macquarie Island (-54.58, } \\
\text { 158.88) } 100-200 \mathrm{~m}\end{array}$ & $\begin{array}{l}\text { tundra lacking woody plants } \\
\text { (closed short herb vegetation) }\end{array}$ & $\begin{array}{l}\text { Poaceae, Acaena, Luzula, } \\
\text { Bryophyta }\end{array}$ & $\begin{array}{l}\text { (mollic) andosols (on pillow } \\
\text { basalt) }\end{array}$ & $\begin{array}{l}\text { Selkirk et al. } \\
1990, \\
\text { Selkirk \& } \\
\text { Adamson } \\
2011\end{array}$ \\
\hline $\begin{array}{l}\text { Subtropical Dry Forest } \\
\text { (SCs): Mediterranean } \\
\text { Division }\end{array}$ & $\begin{array}{l}\text { USA } \\
\text { San Pablo Reservoir, Contra } \\
\text { Costa Co., California (37.92, - } \\
\text { 122.25) } 170 \mathrm{~m}\end{array}$ & woodland & Arbutus menziesii & $\begin{array}{l}\text { chromic luvisols, dystric } \\
\text { fluvisols, mollic gleysols }(60 \text {, } \\
30,10)\end{array}$ & CDMR 2011 \\
\hline $\begin{array}{l}\text { Subtropical Dry Forest } \\
\text { (SCs): Mediterranean } \\
\text { sclerophyllous forests and } \\
\text { scrub }\end{array}$ & $\begin{array}{l}\text { Italy } \\
\text { Tuscany, Arezzo, Alpe de la } \\
\text { Luna }(43.64,12.17) 600-1500 \\
\text { m }\end{array}$ & $\begin{array}{l}\text { coppices and transitory high } \\
\text { forests of broad-leaved trees }\end{array}$ & $\begin{array}{l}\text { Fagus, Crataegus monogyna, } \\
\text { Laburnum alpinum, Quercus cerris, } \\
\text { Sorbus aria, Taxus baccata, Ilex } \\
\text { aquifolium, Carpinus betulus, } \\
\text { Cardamine enneaphyllos }\end{array}$ & $\begin{array}{l}\text { eutric cambisols, haplic } \\
\text { luvisols, rendzic leptosols ( } 45 \text {, } \\
30,25) \text { (sandstones and marls) }\end{array}$ & $\begin{array}{l}\text { Salerni \& } \\
\text { Perini } 2007\end{array}$ \\
\hline
\end{tabular}




\begin{tabular}{|c|c|c|c|c|c|}
\hline Ecozone FAO & Site & Habitat & Vegetation & Soil & Sources* \\
\hline $\begin{array}{l}\text { Subtropical Dry Forest } \\
\text { (SCs): Mediterranean } \\
\text { sclerophyllous forests and } \\
\text { scrub }\end{array}$ & $\begin{array}{l}\text { Portugal } \\
\text { Alentejo, Parque de Natureza de } \\
\text { Noudar }(38.18,-7.04) 215 \mathrm{~m}\end{array}$ & $\begin{array}{l}\text { under Cistus in Quercus } \\
\text { forest }\end{array}$ & $\begin{array}{l}\text { Cistus ladanifer, Quercus } \\
\text { rotundifolia }\end{array}$ & $\begin{array}{l}\text { eutric regosols, eutric } \\
\text { cambisols, dystric regosols, } \\
\text { lithic leptosols, haplic luvisols, } \\
\text { haplic acrisols, rock outcrops } \\
(35,20,15,15,5,5,5)\end{array}$ & $\begin{array}{l}\text { Louro et al. } \\
2009\end{array}$ \\
\hline $\begin{array}{l}\text { Subtropical Dry Forest } \\
\text { (SCs): Mediterranean } \\
\text { sclerophyllous forests and } \\
\text { scrub }\end{array}$ & $\begin{array}{l}\text { Turkey } \\
\text { Giresun, Görele, Ardic (40.95, } \\
39.01) 440 \mathrm{~m}\end{array}$ & on lawns & Poaceae & $\begin{array}{l}\text { orthic acrisols, rankers, humic } \\
\text { cambisols, dystric cambisols, } \\
\text { lithosols }(30,20,20,20,10)\end{array}$ & Sesli 2007 \\
\hline $\begin{array}{l}\text { Subtropical Dry Forest } \\
\text { (SCs): Mediterranean } \\
\text { sclerophyllous forests and } \\
\text { scrub }\end{array}$ & $\begin{array}{l}\text { Turkey } \\
\text { Gümüshane, Kürtün, Kizilot } \\
(40.72,39.09) 730 \mathrm{~m}\end{array}$ & amongst grass & Poaceae & $\begin{array}{l}\text { orthic acrisols, rankers, humic } \\
\text { cambisols, dystric cambisols, } \\
\text { lithosols }(30,20,20,20,10)\end{array}$ & Sesli 2007 \\
\hline $\begin{array}{l}\text { Subtropical Dry Forest } \\
\text { (SCs): Mediterranean } \\
\text { sclerophyllous forests and } \\
\text { scrub }\end{array}$ & $\begin{array}{l}\text { Turkey } \\
\text { Trabzon, Macka, Karakaban } \\
(41.00,39.73) 70 \mathrm{~m}\end{array}$ & in pasture & Poaceae, herbs & $\begin{array}{l}\text { orthic acrisols, rankers, humic } \\
\text { cambisols, dystric cambisols, } \\
\text { lithosols }(30,20,20,20,10)\end{array}$ & Sesli 2007 \\
\hline $\begin{array}{l}\text { Subtropical Dry Forest } \\
\text { (SCs): Swan Coastal Plain } \\
\text { (SWA) }\end{array}$ & $\begin{array}{l}\text { Australia } \\
\text { Perth, Whiteman Park (-31.89, } \\
\text { 115.96) } 5 \mathrm{~m}\end{array}$ & $\begin{array}{l}\text { natural bushland, heathlands, } \\
\text { growing in mosses }\end{array}$ & $\begin{array}{l}\text { Banksia, Eucalyptus, Melaleuca, } \\
\text { Nutsya, Xanthorrhoea, Hibbertia, } \\
\text { Acacia, Verticordia, Bryophyta }\end{array}$ & $\begin{array}{l}\text { dystric regosols, eutric } \\
\text { regosols, cambic arenosols, } \\
\text { calcaric regosols }(50,20,20 \text {, } \\
10)\end{array}$ & $\begin{array}{l}\text { Bougher et } \\
\text { al. } 2006, \text { WP } \\
2011\end{array}$ \\
\hline $\begin{array}{l}\text { Subtropical Humid Forest } \\
\text { (SCf): Northland } \\
\text { Temperate Forest }\end{array}$ & $\begin{array}{l}\text { New Zealand } \\
\text { Waikato, Mt Pirongia, W. Scott } \\
\text { F.R (-38.07, 175.09) 200-280 m }\end{array}$ & broadleaved-podocarp forest & Podocarpus & $\begin{array}{l}\text { dystric cambisols, ochric } \\
\text { andosols }(70,30)\end{array}$ & LRNZ 2011 \\
\hline $\begin{array}{l}\text { Subtropical Humid Forest } \\
\text { (SCf): Northland } \\
\text { Temperate Forest }\end{array}$ & $\begin{array}{l}\text { New Zealand } \\
\text { Wellington, Rimutaka Forest } \\
\text { Park, Orongorongo River track } \\
(-41.36,175.00) 100-400 \mathrm{~m}\end{array}$ & broadleaved-podocarp forest & Podocarpus & $\begin{array}{l}\text { dystric cambisols, rankers }(90, \\
10)\end{array}$ & LRNZ 2011 \\
\hline $\begin{array}{l}\text { Subtropical Humid Forest } \\
\text { (SCf): Northland } \\
\text { Temperate Kauri Forest }\end{array}$ & $\begin{array}{l}\text { New Zealand } \\
\text { Northland, Waipoua State } \\
\text { Forest, Te Matua Ngahere Track } \\
(-35.65,173.56) 100 \mathrm{~m}\end{array}$ & kauri forest & Agathis australis & $\begin{array}{l}\text { humic acrisols, eutric } \\
\text { cambisols, gleyic acrisols }(50 \text {, } \\
20,20)\end{array}$ & LRNZ 2011 \\
\hline $\begin{array}{l}\text { Subtropical Humid Forest } \\
\text { (SCf): Subtropical Hygro- } \\
\text { thermophilous mixed } \\
\text { deciduous broad-leaved } \\
\text { forests }\end{array}$ & $\begin{array}{l}\text { Canaries } \\
\text { La Palma, Los Tilos }(28.79,- \\
\text { 17.80) } 510 \mathrm{~m}\end{array}$ & laurel forest („Laurisilva“) & $\begin{array}{l}\text { Laurus novocanariensis, Persea } \\
\text { indica, Myrica faya, Erica arborea, } \\
\text { Ilex canariensis, Viburnum rigidum, } \\
\text { Hypericum grandifolium, herbs, } \\
\text { ferns, no mosses }\end{array}$ & $\begin{array}{l}\text { chromic luvisols, ochric } \\
\text { andosols, orthic luvisols, } \\
\text { dystric fluvisols, lithosols ( } 40 \text {, } \\
20,20,10,10)\end{array}$ & $\begin{array}{l}\text { Karasch } \\
\text { 2006, Castro } \\
\text { pers. comm. }\end{array}$ \\
\hline
\end{tabular}




\begin{tabular}{|c|c|c|c|c|c|}
\hline Ecozone FAO & Site & Habitat & Vegetation & Soil & Sources* \\
\hline $\begin{array}{l}\text { Subtropical Humid } \\
\text { Forests (SCf): Sydney } \\
\text { Basin }\end{array}$ & $\begin{array}{l}\text { Australia } \\
\text { Sidney, Lane Cove Bushland } \\
\text { Park, core zone along } \\
\text { the Gore Creek catchment (- } \\
33.82,151.18) 15 \mathrm{~m}\end{array}$ & $\begin{array}{l}\text { warm-temperate wet } \\
\text { sclerophyll gallery forest }\end{array}$ & $\begin{array}{l}\text { Eucalyptus, Angophora, } \\
\text { Ceratopetalum apetalum, Poaceae, } \\
\text { Lepidozia }\end{array}$ & $\begin{array}{l}\text { humic podzols, orthic podzols, } \\
\text { gleyic podzols, planosols, } \\
\text { dystric regosols, calcaric } \\
\text { regosols }(30,20,20,10,10,10)\end{array}$ & $\begin{array}{l}\text { Kearney \& } \\
\text { Kearney } \\
\text { 2007, Lepp } \\
2001\end{array}$ \\
\hline $\begin{array}{l}\text { Temperate Continental } \\
\text { Forest (TeDc): Montane } \\
\text { coniferous forests, partly } \\
\text { with broadleaved trees }\end{array}$ & $\begin{array}{l}\text { Switzerland } \\
\text { Merishausen (Uff der Grate, } \\
\text { Gratental) }(47.76,8.62) 570-710 \\
\mathrm{~m}\end{array}$ & $\begin{array}{l}\text { grassland at } \leq 1 \text { swathe per } \\
\text { year }\end{array}$ & $\begin{array}{l}\text { Medicagini falcatea -, Dauco-Salvio } \\
\text {-, Seselio libanotidis - } \\
\text { Mesobrometum }\end{array}$ & $\begin{array}{l}\text { orthic rendzina, eutric cambisol } \\
(60,30,10)\end{array}$ & $\begin{array}{l}\text { Brunner } \\
1987\end{array}$ \\
\hline $\begin{array}{l}\text { Temperate Continental } \\
\text { Forests }(\mathrm{TeDc}): \text { forest } \\
\text { steppes }\end{array}$ & $\begin{array}{l}\text { Germany } \\
\text { Amorbach-West, „Vordere } \\
\text { Äcker“ }(49.65,9.20) 200 \mathrm{~m}\end{array}$ & $\begin{array}{l}\text { mesotrophic, herb-rich sheep } \\
\text { meadow, 2-3 swathes per } \\
\text { year }\end{array}$ & $\begin{array}{l}\text { Rhytidiadelphus squarrosus, } \\
\text { Festuca rubra, Trifolium pratense, } \\
\text { Agrostis capillaris, Leontodon } \\
\text { hispidus, Cirriphyllum piliferum, } \\
\text { Plantago lanceolata, Lotus } \\
\text { corniculatus plus } 36 \text { species with } \\
\text { low abundance }\end{array}$ & $\begin{array}{l}\text { dystric cambisols, leptic } \\
\text { podzols, stagno-gleyic } \\
\text { cambisols }(60,20,20)\end{array}$ & $\begin{array}{l}\text { Kaiser pers. } \\
\text { comm., } \\
\text { Halbwachs } \\
\text { pers.obs. }\end{array}$ \\
\hline $\begin{array}{l}\text { Temperate Continental } \\
\text { Forests }(\mathrm{TeDc}) \text { : forest } \\
\text { steppes }\end{array}$ & $\begin{array}{l}\text { Germany } \\
\text { Hartschimmel / Ammersee, } \\
\text { Goaslweide }(47.94,11.18) 720 \\
\mathrm{~m}\end{array}$ & $\begin{array}{l}\text { oligotrophic, extant } \\
\text { woodland pasture including a } \\
\text { calcerous fen }\end{array}$ & $\begin{array}{l}\text { Agrostis capillaris, Anemone } \\
\text { nemorosa, Brachypodium rupestre, } \\
\text { Briza media, Bromus erectus, } \\
\text { Calluna vulgaris, Cynosurus } \\
\text { cristatus, Carex (5 species), } \\
\text { Festtuca (4 species), Carlina } \\
\text { acaulis, Centaurea scabiosa, } \\
\text { Dactylis glomerata, Danthonia } \\
\text { decumbens, Hippocrepis comosa } \\
\text { plus roughly } 300 \text { more species, } \\
\text { including trees such as Quercus } \\
\text { robur, Fagus sylvatica, Fraxinus } \\
\text { excelsior, Abies alba }\end{array}$ & $\begin{array}{l}\text { orthic luvisols, eutric } \\
\text { cambisols, eutric regosols, } \\
\text { stagno-gleyic luvisols }(70,20 \text {, } \\
5,5)\end{array}$ & $\begin{array}{l}\text { Karasch } \\
2001\end{array}$ \\
\hline $\begin{array}{l}\text { Temperate Continental } \\
\text { Forests }(\mathrm{TeDc}) \text { : forest } \\
\text { steppes }\end{array}$ & $\begin{array}{l}\text { UK } \\
\text { Cardiff, Llanishen Reservoir } \\
\text { Embankment, Radyr Cricket } \\
\text { Ground / junction fields (51.52, - } \\
\text { 3.18) } 20 \mathrm{~m}\end{array}$ & grassland & Poaceae, herbs & urban & $\begin{array}{l}\text { Grantham et } \\
\text { al. } 2009\end{array}$ \\
\hline $\begin{array}{l}\text { Temperate Continental } \\
\text { Forests }(\mathrm{TeDc}) \text { : forest } \\
\text { steppes }\end{array}$ & $\begin{array}{l}\text { UK } \\
\text { Wales, Dinefwr Park (51.88, - } \\
\text { 4.00) } 65 \mathrm{~m}\end{array}$ & estate parkland & Poaceae & $\begin{array}{l}\text { dystric cambisols, ferric } \\
\text { podzols, dystric gleysols, } \\
\text { umbric leptosols, rock outcrops } \\
(65,10,10,10,5)\end{array}$ & $\begin{array}{l}\text { Rotheroe } \\
1999\end{array}$ \\
\hline
\end{tabular}




\begin{tabular}{|c|c|c|c|c|c|}
\hline Ecozone FAO & Site & Habitat & Vegetation & Soil & Sources* \\
\hline $\begin{array}{l}\text { Temperate mountain } \\
\text { systems (TM) }\end{array}$ & $\begin{array}{l}\text { Nepal } \\
\text { Solu-Khumbu, Khunde, } \\
\text { Sagarmatha National Park } \\
(27.82,86.71) 3900 \mathrm{~m}\end{array}$ & $\begin{array}{l}\text { on moist soil in birch - } \\
\text { rhododendron forest }\end{array}$ & $\begin{array}{l}\text { Betula utilis, Rhododendron } \\
\text { campanulatum, } R \text {. campylocarpum }\end{array}$ & $\begin{array}{l}\text { lithosols, rock outcrops, } \\
\text { rankers, humic cambisols }(25, \\
25,25,25)\end{array}$ & Giri 2007 \\
\hline $\begin{array}{l}\text { Temperate Mountain } \\
\text { Systems (TM): Alpine } \\
\text { vegetation and } \\
\text { Montane steppes }\end{array}$ & $\begin{array}{l}\text { Switzerland } \\
\text { Engadin, Swiss National Park, } \\
\text { Alp Schera }(46.64,10.19) 2000 \\
\text { m }\end{array}$ & $\begin{array}{l}\text { clearings in alpine forests, } \\
\text { alpine grassland }\end{array}$ & Pinus, Poaceae, herbs & $\begin{array}{l}\text { orthic podzols, dystric } \\
\text { podzoluvisols, ranker }(45,40 \text {, } \\
15)\end{array}$ & Favre 1960 \\
\hline $\begin{array}{l}\text { Temperate Mountain } \\
\text { Systems (TM): Alpine } \\
\text { vegetation and } \\
\text { Montane steppes }\end{array}$ & $\begin{array}{l}\text { Switzerland } \\
\text { Engadin, Swiss National Park, } \\
\text { Fuorn, Murteras da Grimmels } \\
(46.68,10.19) 2500 \mathrm{~m}\end{array}$ & alpine grassland & Poaceae, herbs & $\begin{array}{l}\text { orthic podzols, dystric } \\
\text { podzoluvisols, ranker }(45,40 \text {, } \\
15)\end{array}$ & Favre 1960 \\
\hline $\begin{array}{l}\text { Temperate Mountain } \\
\text { Systems (TM): Alpine } \\
\text { vegetation and } \\
\text { Montane steppes }\end{array}$ & $\begin{array}{l}\text { Switzerland } \\
\text { Engadin, Swiss National Park, } \\
\text { Mot dal Gajer }(46.71,10.33) \\
2600 \mathrm{~m}\end{array}$ & $\begin{array}{l}\text { climbing beyond tree-line, } \\
\text { alpine grassland }\end{array}$ & Poaceae, herbs & lithosol, dystric regosol $(60,40)$ & Favre 1960 \\
\hline $\begin{array}{l}\text { Temperate Mountain } \\
\text { Systems (TM): Alpine } \\
\text { vegetation and } \\
\text { Montane steppes }\end{array}$ & $\begin{array}{l}\text { Switzerland } \\
\text { Engadin, Swiss National Park, } \\
\text { Piz Starlex }(46.66,10.38) 2650 \\
\text { m }\end{array}$ & alpine grassland & Poaceae, herbs & $\begin{array}{l}\text { lithosols, dystric regosols ( } 60 \text {, } \\
40)\end{array}$ & Favre 1960 \\
\hline $\begin{array}{l}\text { Temperate Mountain } \\
\text { Systems (TM): Alpine } \\
\text { vegetation and } \\
\text { Montane steppes }\end{array}$ & $\begin{array}{l}\text { Switzerland } \\
\text { Engadin, Swiss National Park, } \\
\text { Plateau de San Jon }(46.79 \text {, } \\
\text { 10.31) } 1450 \text { (2550) m }\end{array}$ & $\begin{array}{l}\text { grassy plateau (climbing } \\
\text { beyond tree-line), no soil } \\
\text { preferences, alpine grassland }\end{array}$ & Poaceae, herbs & $\begin{array}{l}\text { orthic podzols, dystric } \\
\text { podzoluvisols, ranker }(45,40 \text {, } \\
15)\end{array}$ & Favre 1960 \\
\hline $\begin{array}{l}\text { Temperate Mountain } \\
\text { Systems (TM): Alpine } \\
\text { vegetation and } \\
\text { Montane steppes }\end{array}$ & $\begin{array}{l}\text { Switzerland } \\
\text { Engadin, Swiss National Park, } \\
\text { S-chanf, Alp Griatschouls } \\
(46.63,9.97) 1700 \mathrm{~m}\end{array}$ & dry gravelly slope & $\begin{array}{l}\text { Calamintha alpina, Petasites } \\
\text { paradoxus, Gypsophila repens }\end{array}$ & $\begin{array}{l}\text { orthic podzols, dystric } \\
\text { podzoluvisols, ranker }(45,40 \text {, } \\
15)\end{array}$ & Favre 1960 \\
\hline $\begin{array}{l}\text { Temperate Mountain } \\
\text { Systems (TM): Alpine } \\
\text { vegetation and } \\
\text { Montane steppes }\end{array}$ & $\begin{array}{l}\text { Switzerland } \\
\text { Engadin, Swiss National Park, } \\
\text { Stabelchod }(46.66,10.24) 1900 \\
\text { m }\end{array}$ & $\begin{array}{l}\text { alpine coniferous forests, } \\
\text { alpine grassland }\end{array}$ & Pinus mиgo, Poaceae, herbs & $\begin{array}{l}\text { orthic podzols, dystric } \\
\text { podzoluvisols, ranker }(45,40 \text {, } \\
15)\end{array}$ & Favre 1960 \\
\hline $\begin{array}{l}\text { Temperate Mountain } \\
\text { Systems (TM): Alpine } \\
\text { vegetation and } \\
\text { Montane steppes }\end{array}$ & $\begin{array}{l}\text { Switzerland } \\
\text { Engadin, Swiss National Park, } \\
\text { Susch, Sandögna }(46.73,10.08) \\
1450 \mathrm{~m}\end{array}$ & alpine grassland & Poaceae & $\begin{array}{l}\text { dystric podzoluvisols, dystric } \\
\text { cambisols, orthic podzols ( } 40 \text {, } \\
30,30)\end{array}$ & Favre 1960 \\
\hline
\end{tabular}




\begin{tabular}{|c|c|c|c|c|c|}
\hline Ecozone FAO & Site & Habitat & Vegetation & Soil & Sources* \\
\hline Temperate Mountain & Switzerland & beyond treeline, alpine & Poaceae, herbs & orthic rendzina, calcaric & Favre 1960 \\
\hline $\begin{array}{l}\text { Systems }(\mathrm{TM}) \text { : Alpine } \\
\text { vegetation and }\end{array}$ & $\begin{array}{l}\text { Engadin, Swiss National Park, } \\
\text { Val Laschadura }(46.70,10.16)\end{array}$ & grassland & & $\begin{array}{l}\text { gleysols, humic cambisol, } \\
\text { eutric podzoluvisols }(30,30 \text {, }\end{array}$ & \\
\hline Montane steppes & $2350 \mathrm{~m}$ & & & $20,20)$ & \\
\hline Temperate Mountain & Switzerland & dry calcerous gravelly slope & Calamintha alpina, Petasites & orthic podzols, dystric & Favre 1960 \\
\hline $\begin{array}{l}\text { Systems (TM): Alpine } \\
\text { vegetation and }\end{array}$ & $\begin{array}{l}\text { Engadin, Swiss National Park, } \\
\text { Val Trupchun }(46.62,10.02)\end{array}$ & & paradoxus, Gypsophila repens & $\begin{array}{l}\text { podzoluvisols, ranker }(45,40 \text {, } \\
15)\end{array}$ & \\
\hline Montane steppes & $1800 \mathrm{~m}$ & & & & \\
\hline Temperate Mountain & Australia & Tasmanian bolster heath & Dracophyllum minimum, Donatia & chromic cambisols, regosols, & McMullan- \\
\hline $\begin{array}{l}\text { Systems (TM): Australian } \\
\text { Alps }\end{array}$ & $\begin{array}{l}\text { Tasmania, Mt. Field, Newdegate } \\
\text { Pass }(-42.65,146.56) 1280 \mathrm{~m}\end{array}$ & & $\begin{array}{l}\text { novae-zelandiae, lilies, daisies, } \\
\text { grasses, herbs, sedges }\end{array}$ & $\begin{array}{l}\text { lithosols, dystric histosols, } \\
\text { humic cambisols }(40,20,20 \text {, } \\
10,10)\end{array}$ & $\begin{array}{l}\text { Fisher et al. } \\
2003 \text {, FPA } \\
2007\end{array}$ \\
\hline $\begin{array}{l}\text { Temperate Oceanic Forest } \\
\text { (TeDo) }\end{array}$ & $\begin{array}{l}\text { Ireland } \\
\text { The Curragh, Kildare (53.16, - } \\
6.83) 100 \mathrm{~m}\end{array}$ & acidic, semi-natural grassland & Poaceae, herbs, Ulex & $\begin{array}{l}\text { orthic luvisol, eutric cambisol, } \\
\text { eutric gleysols, terric histosols } \\
(50,25,20,5)\end{array}$ & $\begin{array}{l}\text { McHugh et } \\
\text { al. } 2001\end{array}$ \\
\hline $\begin{array}{l}\text { Temperate Oceanic } \\
\text { Forests (TeDo): Southland } \\
\text { Temperate Forest }\end{array}$ & $\begin{array}{l}\text { New Zealand } \\
\text { Nelson, Murchison cemetery (- } \\
41.80,172.34) 150 \mathrm{~m}\end{array}$ & cemetery & grass & $\begin{array}{l}\text { dystric cambisols, orthic } \\
\text { podzols, lithosols }(60,30,10)\end{array}$ & LRNZ 2011 \\
\hline $\begin{array}{l}\text { Temperate Oceanic } \\
\text { Forests (TeDo): Southland } \\
\text { Temperate Forest }\end{array}$ & $\begin{array}{l}\text { New Zealand } \\
\text { North Canterbury, Glentui Bush } \\
(-43.20,172.25) 400 \mathrm{~m}\end{array}$ & beech-broadleaved forest & Nothofagus & $\begin{array}{l}\text { eutric cambisols, orthic } \\
\text { luvisols, gleyic luvisols }(60,20 \text {, } \\
20)\end{array}$ & $\begin{array}{l}\text { LRNZ 2011, } \\
\text { Te Ara 2011 }\end{array}$ \\
\hline $\begin{array}{l}\text { Temperate Steppe } \\
\text { (TeBSk): coastal dune } \\
\text { vegetation }\end{array}$ & $\begin{array}{l}\text { UK } \\
\text { Aberdeenshire, Blairton Links } \\
(57.26,-2.04) 10 \mathrm{~m}\end{array}$ & $\begin{array}{l}\text { fixed acidic dune with } \\
\text { grassland }\end{array}$ & $\begin{array}{l}\text { Salix repens, Poaceae a.o. } \\
\text { Corynephorus canescens, } \\
\text { Empetrum nigrum }\end{array}$ & dystric regosols, sand & $\begin{array}{l}\text { Holden } \\
2007, \mathrm{BFN} \\
2004\end{array}$ \\
\hline $\begin{array}{l}\text { Tropical Mountain } \\
\text { Systems (TM): Albertine } \\
\text { Rift Montane Forest } \\
\text { Ecoregion }\end{array}$ & $\begin{array}{l}\text { Congo } \\
\text { Bukavu, slopes of Mt. Kahuzi (- } \\
2.47,28.80) 1850 \mathrm{~m}\end{array}$ & savannah & Hyparrhenia & $\begin{array}{l}\text { humic ferralsols, humic nitisols } \\
(60,40)\end{array}$ & $\begin{array}{l}\text { McGINLEY } \\
2009\end{array}$ \\
\hline $\begin{array}{l}\text { Tropical mountain } \\
\text { systems (TM): } \\
\text { Hispaniolan Pine Forest }\end{array}$ & $\begin{array}{l}\text { Dominican Republic } \\
\text { Santiago, San José de Las Matas } \\
(19.34,-70.94) 520 \mathrm{~m}\end{array}$ & pine forest, on soil & Pinus occidentalis & $\begin{array}{l}\text { eutric cambisols, haplic } \\
\text { acrisols, chromic cambisols, } \\
\text { eutric leptosols }(40,15,30,15)\end{array}$ & TOA 2011b \\
\hline
\end{tabular}




\begin{tabular}{|c|c|c|c|c|c|}
\hline Ecozone FAO & Site & Habitat & Vegetation & Soil & Sources* \\
\hline $\begin{array}{l}\text { Tropical Mountain } \\
\text { Systems (TM): Tropical } \\
\text { Central American Sierra } \\
\text { Madre and Chiapas } \\
\text { highlands }\end{array}$ & $\begin{array}{l}\text { Mexico } \\
\text { Chiapas, municipio de } \\
\text { Ocozocoautla de Espinosa, } \\
\text { Parque Educativo Laguna } \\
\text { Bélgica }(16.88,-93.46) 1000 \mathrm{~m}\end{array}$ & $\begin{array}{l}\text { (Acahual) Selva Mediana } \\
\text { subperennifolia (disturbed } \\
\text { evergreen seasonal forest) }\end{array}$ & $\begin{array}{l}\text { Brosimum alicastrum, Bursera } \\
\text { simaruba, Cedrela odorata, } \\
\text { Chamaedorea ernesti-augusti, } \\
\text { Cordia alliodora, Ficus involuta, } \\
\text { Pseudolmedia oxyphyllaria, } \\
\text { Terminalia amazonia, Cecropia, } \\
\text { Croton draco, Heliocarpus } \\
\text { donnellsmithii, Piper auritum, } \\
\text { Trema micrantha, Trichospermum } \\
\text { mexicanum }\end{array}$ & $\begin{array}{l}\text { haplic luvisols, rendzic } \\
\text { leptosols }(70,30)\end{array}$ & $\begin{array}{l}\text { Chanona- } \\
\text { Gomez et al. } \\
2007, \\
\text { Escobar- } \\
\text { Ocampo \& } \\
\text { Ochoa- } \\
\text { Gaona } 2007\end{array}$ \\
\hline $\begin{array}{l}\text { Tropical Mountain } \\
\text { Systems (TM): Tropical } \\
\text { medium elevation } \\
\text { subhumid }\end{array}$ & $\begin{array}{l}\text { Kenya } \\
\text { Mt. Kenya, Naro Moru track (- } \\
0.18,37.15) 2450 \mathrm{~m}\end{array}$ & $\begin{array}{l}\text { Podocarpus forest, plant } \\
\text { debris }\end{array}$ & Podocarpus & $\begin{array}{l}\text { humic lithosols, umbric } \\
\text { andosols }(60,40)\end{array}$ & Kost 2002 \\
\hline Tropical rain forest (TAr) & $\begin{array}{l}\text { Hawaiian Islands } \\
\text { Moloka'i, Kamakou Forest } \\
\text { Preserve, Kamoku Flats (21.12, - } \\
\text { 156.92) } 1110 \mathrm{~m}\end{array}$ & $\begin{array}{l}\text { in soil under introduced } \\
\text { Monterey Cypress }\end{array}$ & Cupressus macrocarpa & $\begin{array}{l}\text { lithosols, humic andosols (50, } \\
50)\end{array}$ & $\begin{array}{l}\text { Desjardin \& } \\
\text { Hemmes } \\
1997\end{array}$ \\
\hline $\begin{array}{l}\text { Tropical Rain Forest } \\
\text { (TAr): Tropical moist } \\
\text { forest (marine association) }\end{array}$ & $\begin{array}{l}\text { Costa Rica } \\
\text { Cocos Island, Cerro Iglesias } \\
(5.53,-87.08) 280(?) \mathrm{m}\end{array}$ & $\begin{array}{l}\text { moist tropical forest, among } \\
\text { mosses }\end{array}$ & Bryophyta & $\begin{array}{l}\text { humic lithosols, dystric } \\
\text { gleysols, ferralic cambisols ( } 50 \text {, } \\
25,25)\end{array}$ & $\begin{array}{l}\text { Gómez } \\
1983, \\
\text { Damobrosio } \\
2001\end{array}$ \\
\hline
\end{tabular}


Due to the lack of comparable records about soil properties, we had to rely on the FAO World reference base for soil resources (IUSS 2006) for the classification of the soil types, and on the Harmonized World Soil Database (FAO \& ISRIC 2009) for the identification of the soil types. The latter database works with geographical coordinates for pinpointing any location worldwide with a precision of two decimals $(\mathrm{ca} .1 .1 \mathrm{~km})$. From the findings we extracted a measure about the soil types waxcaps prefer. For this we calculated a preference index by scaling the ratio between the number of sites and the global surface area of a specific soil type between 0 and 100).

\section{Results and Discussion}

As is the case with most biodiversity distribution data there is a bias towards Europe (Fig. 1), and this needs to be kept in mind when assessing our quantitative analyses.

The term Hygrocybe is widely used especially in Europe to refer to the waxcap species found in grassland, as described by Boertmann (2010). However, it is now recognised that this genus is polyphyletic with members of the component taxa spread across the family Hygrophoraceae (cf. Seitzman et al. 2011), a recognition that is not reflected by the results of our analysis. Since there is a lack of fungal molecular data corresponding to the sources investigated, we were not able to look into genetic variations of the selected taxa. It would theoretically be desirable to match molecular data with specific sites. Practically the considerable effort to generate DNA-based data from all the specimens mentioned by our sources would probably not pay off, because the overall trends found within the realm of this review would probably not be affected by genotypic variations.

The comparison of the 65 waxcap sites ranging from the tropical to the polar zones, where one or more of the 16 waxcap species occur, show a broad ecological amplitude (Table 1). For temperate habitats this has already been shown by Bresinsky (2008: 47) who recorded a distribution over almost all habitats in Germany. All 16 species occurred in temperate and boreal habitats, 7 at polar, 9 at subtropical and 3 at tropical locations. H. conica and H. miniata show the greatest latitudinal distribution (poles to tropics), whereas H. chlorophana and H. mucronella are restricted to temperate and boreal climates (Fig. 3).

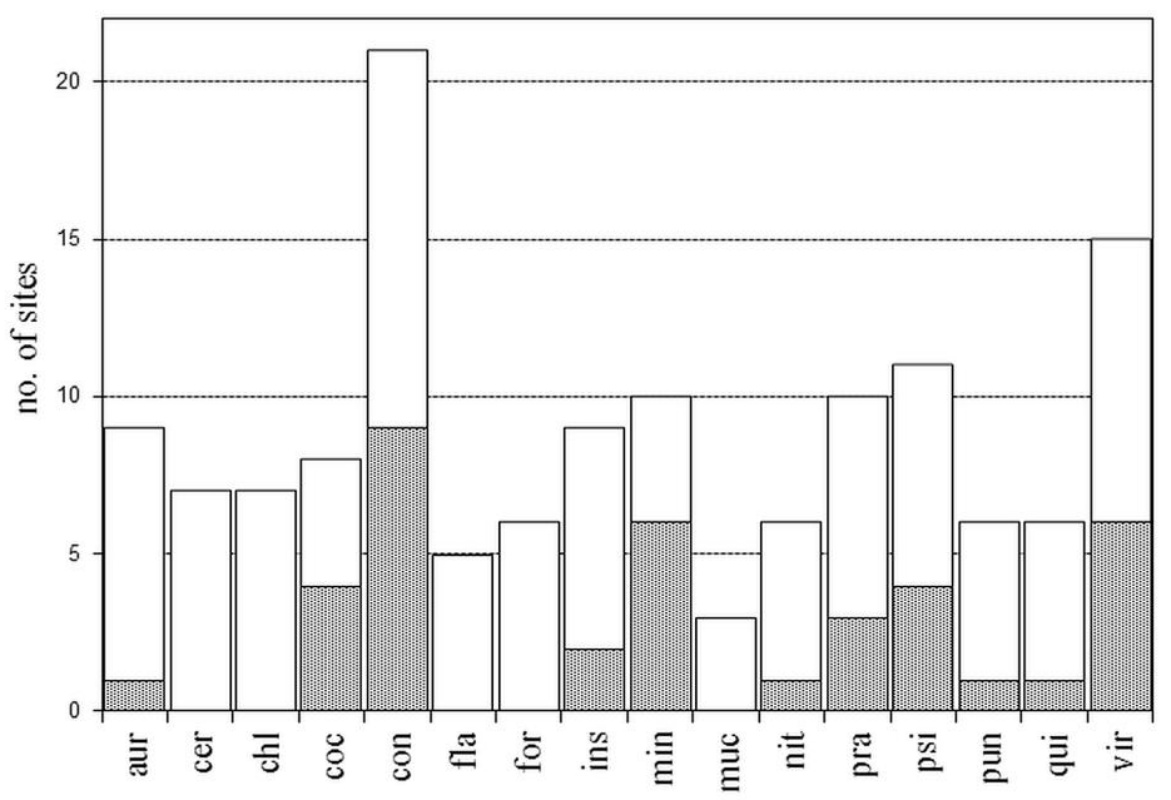

Fig. 3 - Ocurrence of the 16 Hygrocybe species in woodland and grassland. The y-axis shows the number of sites, the x-axis the species, both as defined in our data set. Shaded: woodland, unshaded: grassland. Species abbreviations: aur - aurantiosplendens, cer - ceracea, chl chlorophana, coc - coccinea, con - conica, fla - flavipes, for - fornicata, ins - insipida, min miniata, muc - mucronella, nit - nitrata, pra - pratensis, psi - psittacina, pun - punicea, qui quieta, vir - virginea. 
Waxcaps occur in association with a great variety of plant communities and plant species (table 1). Looking at the distribution in forests relative to grass/heathland (Table 2, Fig. 3), a diverse species-related pattern emerges from the locations investigated. The overall distribution between forests and grass/heathland of the 65 locations follows a proportion of ca. 1:2.6 in favour of the latter. The internet image analysis arrived at comparable results (fig. 4)

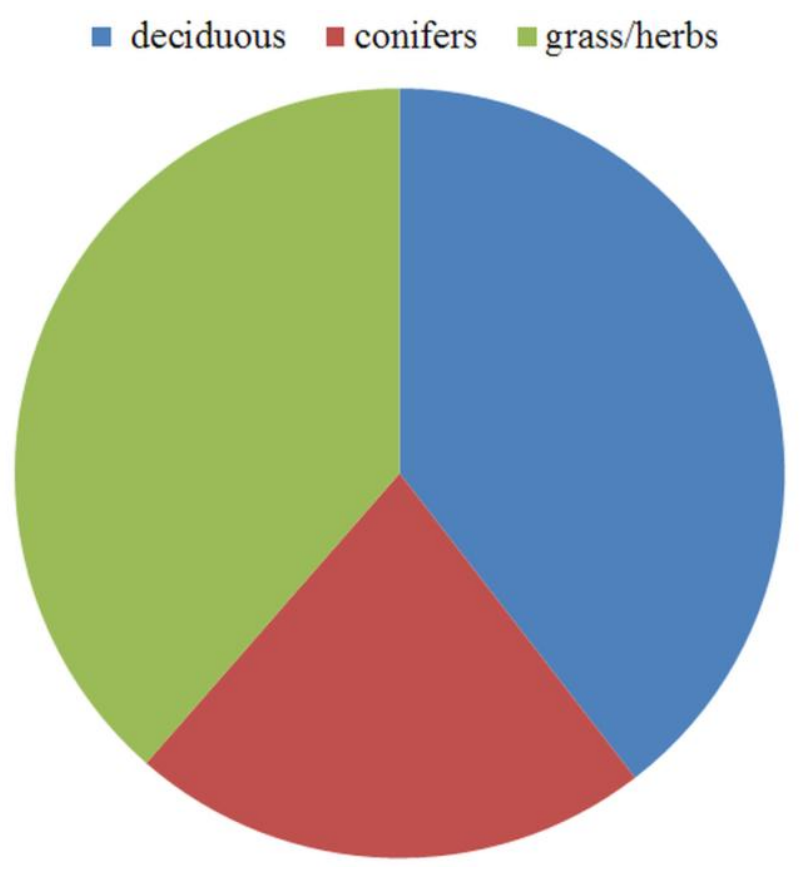

Fig. 4 - Total distribution of the major vegetation types with images of waxcaps out of the 16 selected taxa in Europe and North America. The distribution between forests and grass/heathland follows in Europe a proportion of ca. 1:4.1, in North America the proportion is reversed with ca. $6.5: 1$. In addition, the analysis showed associations with bryophytes for ca. $50 \%$ of the images

Table 2 - Ecozone ranges of the 16 Hygrocybe species in our data set. The ecozones in the first column on the left are defined according to FAO (2001). The second column shows the number of sites in each ecozone. Species abbreviations in the column headers: aur - aurantiosplendens, cer ceracea, chl - chlorophana, coc - coccinea, con - conica, fla - flavipes, for - fornicata, ins insipida, min - miniata, muc - mucronella, nit - nitrata, pra - pratensis, psi - psittacina, pun punicea, qui - quieta, vir - virginea.

\begin{tabular}{|c|c|c|c|c|c|c|c|c|c|c|c|c|c|c|c|c|c|}
\hline & $\stackrel{\mathscr{E}}{\mathscr{E}}$ & $\grave{\Xi}$ & ذे & $\approx$ & 巳 & ธิ & $\stackrel{\overbrace{}}{>}$ & $\grave{s}$ & $\cong$ & : & $\stackrel{\Xi}{\Xi}$ & $\stackrel{\Xi}{\Xi}$ & 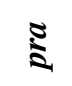 & $\bar{\vdots}$ & $\stackrel{\Xi}{\Sigma}$ & $\widehat{\Xi}$ & $\lesssim$ \\
\hline Tropical rain forest & 2 & & & & & $\mathrm{x}$ & & & & & & & & & & & \\
\hline Tropical mountain systems & 4 & & & & & $\mathrm{x}$ & & & & $\mathrm{x}$ & & & & & & & \\
\hline Subtropical humid forest & 14 & & & & $\mathrm{x}$ & $\mathrm{x}$ & & & $\mathrm{x}$ & $\mathrm{x}$ & & $\mathrm{x}$ & $\mathrm{x}$ & $\mathrm{x}$ & & $\mathrm{x}$ & $\mathrm{x}$ \\
\hline Subtropical dry forest & 12 & & & & & $\mathrm{x}$ & & & & $\mathrm{x}$ & & & $\mathrm{x}$ & $\mathrm{x}$ & & & $\mathrm{x}$ \\
\hline Temperate oceanic forest & 7 & $\mathrm{x}$ & & & & $\mathrm{x}$ & & $\mathrm{x}$ & & $\mathrm{x}$ & & $\mathrm{x}$ & & & $\mathrm{x}$ & $\mathrm{x}$ & \\
\hline Temperate continental forest & 9 & & $\mathrm{x}$ & $\mathrm{x}$ & & $\mathrm{x}$ & $\mathrm{x}$ & & & & $\mathrm{x}$ & & $\mathrm{x}$ & $\mathrm{x}$ & & & $\mathrm{x}$ \\
\hline Temperate steppe & 6 & & $\mathrm{x}$ & & & $\mathrm{x}$ & & & $\mathrm{x}$ & & & & $\mathrm{x}$ & $\mathrm{x}$ & & & $\mathrm{x}$ \\
\hline Temperate mountain systems & 16 & $\mathrm{X}$ & & $\mathrm{x}$ & $\mathrm{x}$ & $\mathrm{x}$ & & $\mathrm{x}$ & $\mathrm{x}$ & $\mathrm{x}$ & & $\mathrm{x}$ & $\mathrm{x}$ & $\mathrm{x}$ & $\mathrm{x}$ & $\mathrm{x}$ & $\mathrm{x}$ \\
\hline Boreal coniferous forest & 4 & & & & & $\mathrm{x}$ & & & & & & & & $\mathrm{x}$ & $\mathrm{x}$ & & $\mathrm{x}$ \\
\hline Boreal tundra woodland & 1 & & & & & & & & & $\mathrm{x}$ & & & & & & & \\
\hline Boreal mountain systems & 16 & $\mathrm{x}$ & $\mathrm{x}$ & $\mathrm{x}$ & $\mathrm{x}$ & $\mathrm{x}$ & $\mathrm{x}$ & $\mathrm{x}$ & $\mathrm{x}$ & $\mathrm{x}$ & $\mathrm{x}$ & $\mathrm{x}$ & $\mathrm{x}$ & $\mathrm{x}$ & $\mathrm{x}$ & $\mathrm{x}$ & $\mathrm{x}$ \\
\hline Polar & 9 & & $\mathrm{x}$ & & & $\mathrm{x}$ & & & $\mathrm{x}$ & $\mathrm{x}$ & & & $\mathrm{x}$ & $\mathrm{x}$ & & & $\mathrm{x}$ \\
\hline
\end{tabular}


The diversity of waxcaps and of the associated vegetation appear not to be connected (cf. Öster 2006, 2008). The notion that poor grassland is an ersatz habitat for forests in Europe seems to be plausible, at first glance. In North America most waxcap locations are reported to be forests (e.g. Hesler \& Smith 1963: 32), contrary to many European countries. But it should be noted that this can partly be attributed to an under-sampling of grassland in the USA (Griffith \& Roderick 2008). Also, one important difference between the two geographical areas concerns the eutrophication status. In North America vast old-growth forest areas still have a natural or semi-natural status (FAO 2006; Greenpeace 2006). In Central Europe most forested areas have undergone intense management activities and suffer from soluble nitrogen, phosphorus and sulphur deposition, leading to eutrophication and acidification (Lorenz et al. 2006, Simpson et al. 2006). Moreover, there is no reason to assume that in European forests waxcap mycelium is present below ground and that the fruiting is inhibited at the same time. One should remember that open woodland and vast areas of grassland existed after the last glaciation, all natural habitats where waxcaps could have thrived. Ungulates kept the vegetation cover short (Svenning 2002, Kirby 2004, Johnson 2009), which encourages the fruiting of waxcaps, as grazing or mowing grassland does (Griffith et al. 2011). An obligate association with mosses can be excluded, because Hygrocybe species colonise moss-free habitats according to our findings, e.g. the laurel forests on La Palma (see also fig. 2). The often observed co-occurrence of waxcaps and mosses could be due to the fact that moss also have a preference for low nutrient soils and an absence of rank vegetation.

The extremely broad spectrum of the vegetation associated with waxcaps (see table 1) and the fact that they occur in habitats with low or high diversity of vegetation, clearly show the independence of waxcaps from specific plant taxa or communities.

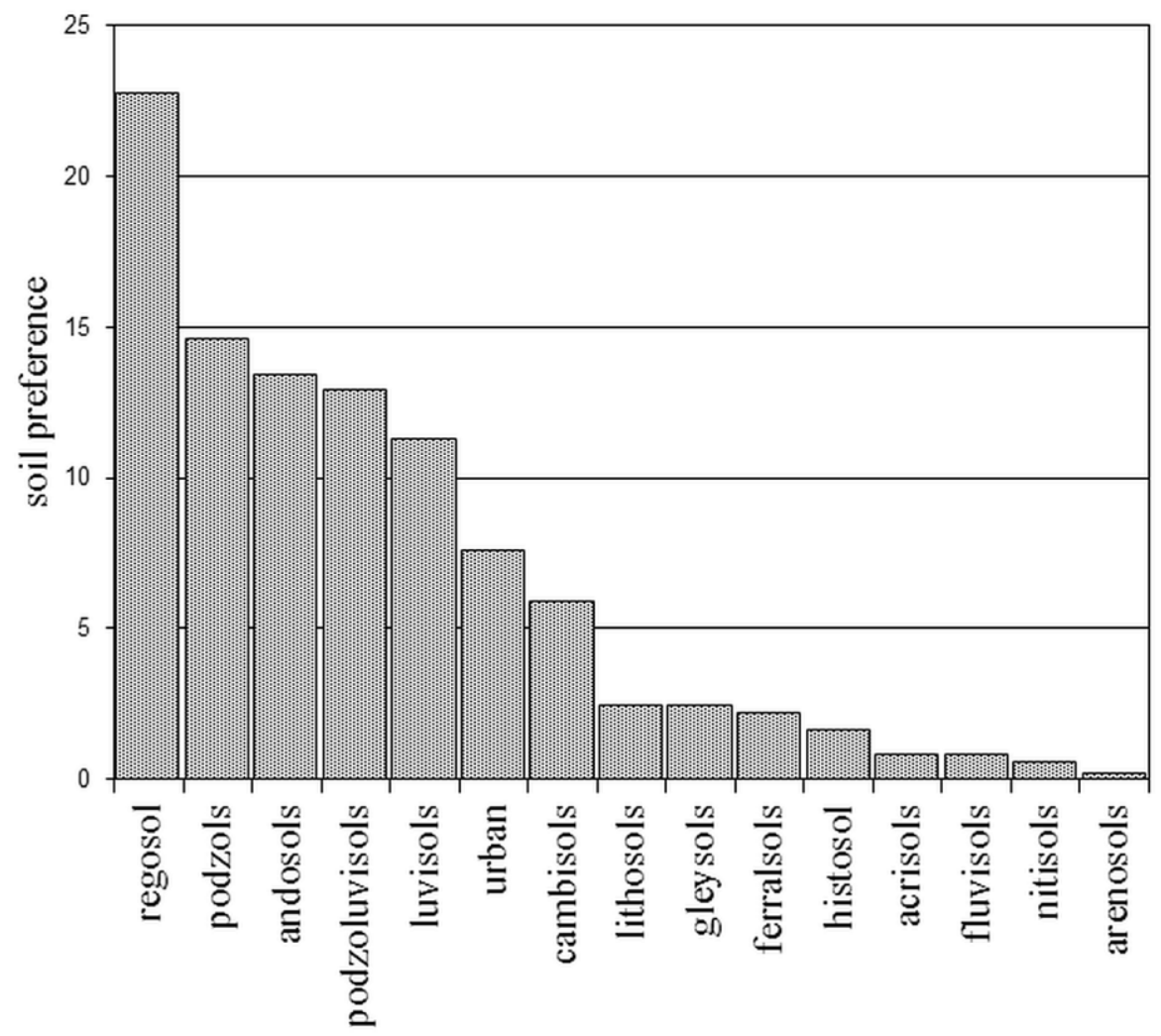

Fig. 5 - Soil preferences of the 16 Hygrocybe species in our data set. The y-axis shows the preferences weighed by the land areas covered by the soil types on the x-axis (source: FAO 2006).

Regosols, podzols, andosols, podzoluvisols and luvisols were the preferred soil types (see Fig. 5). More than two thirds of the waxcap sites have soils of low fertility (dystric). Looking at the 
specific soil requirements of the waxcaps investigated, $H$. conica, $H$. virginea and $H$. psittacina appear to be able to cope with a great variety of soil types.

Most restricted are H. flavipes, mucronella and quieta (Fig. 6). We are aware that the FAO World reference base for soil resources (IUSS 2006) provides a limited resolution which does not reflect possible small-scale variations. Nevertheless, the results appear to be in line with previous appraisals.

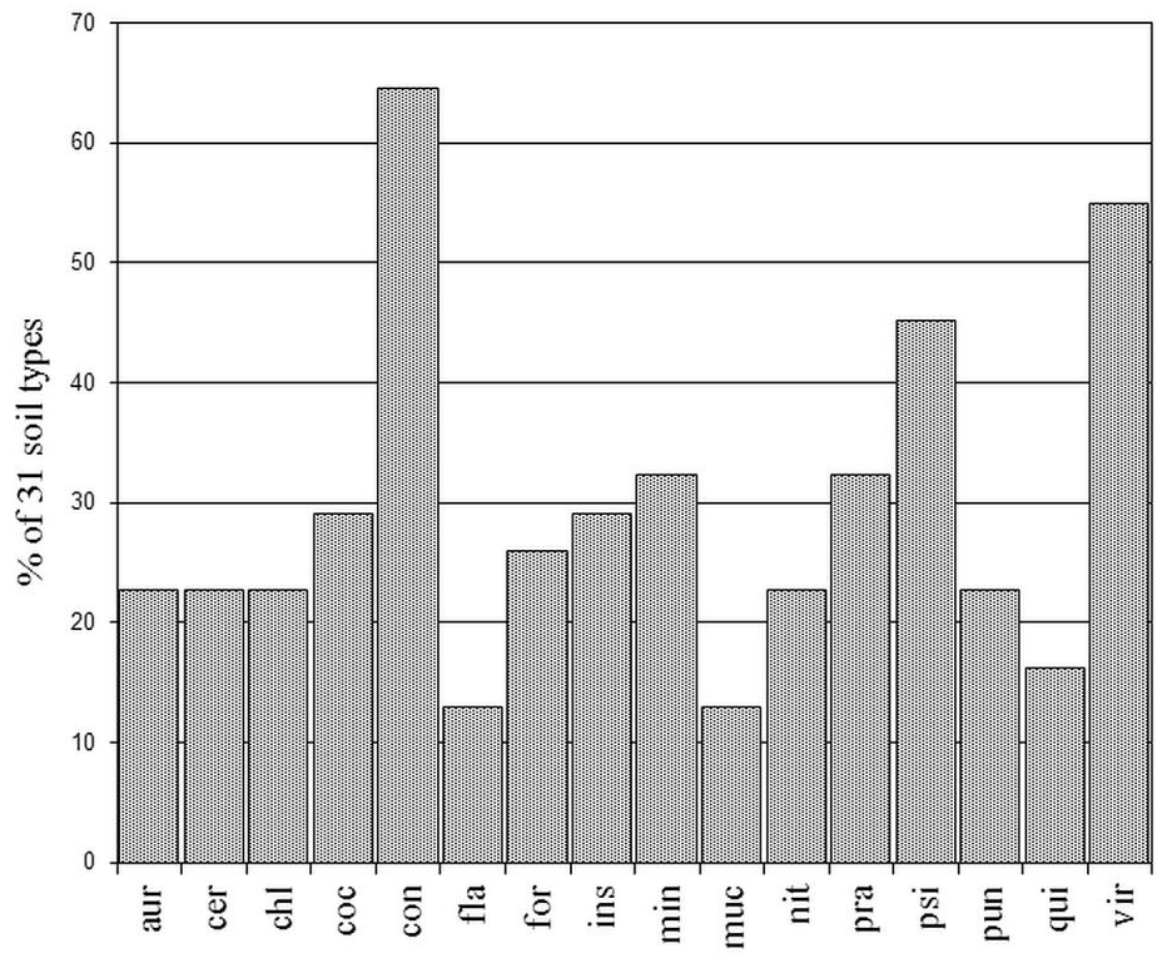

Fig. 6 - Range of soil types by Hygrocybe species. Percentage of the 31 FAO soil types identified (y-axis) where the 16 Hygrocybe species of our data set (x-axis) were found. Species abbreviations: aur - aurantiosplendens, cer - ceracea, chl - chlorophana, coc - coccinea, con - conica, fla flavipes, for - fornicata, ins - insipida, min - miniata, muc - mucronella, nit - nitrata, pra pratensis, psi - psittacina, pun - punicea, qui - quieta, vir - virginea.

The high tolerance of $H$. conica, $H$. virginea and $H$. psittacina in regard to soil types may be the main reason for their widespread distribution. But overall the selected Hygrocybe species show a marked preference for regosols and other dystric soils. Some species (e.g. H. conica and $H$. virginea), often appear as first waxcap species in formerly disturbed habitats, such as dunes (cf. Holden 2007), new highway embankments and green roofs (authors' observations), and roadsides (Beisenherz 2000).

Our review thus confirms at a broader ecological scale the suggestions of other authors (e.g. Griffith et al. 2002, Young 2005, Boertmann 2010: 19) that low fertility, namely in terms of mineralised phosphorus and nitrogen levels, is a prerequisite for most of the 16 waxcap species. Soil organic matter, including humic compounds, seems not to have discriminating effects.

\section{Nutritional considerations}

The small scale associations of several Hygrocybe species (Schweers 1949), also observed by the authors (data not shown), indicate a non-combative lifestyle (cohabitation, see Tuininga 2005). On the other side, the persistence of waxcaps at established, stable sites as often observed by the authors is more typical for a combative c-strategy. The appearance at primary succession sites, such as dunes, new highway embankments and green roofs favours an r-selected strategy. The 
occurrence of waxcaps under extreme environmental conditions would be an expression of a stresstolerant lifestyle (s-selected, see Andrews 1992). These seemingly contradictory traits may be an indication of a high adaptability by changing strategies (Pugh \& Boddy 1988) and/or of the use of a widely distributed nutritional source.

Most of the species in our data set prefer grass- or heathland over woodland, which may be interpreted as avoidance of ectomycorrhizal fungi (hosts) which is corroborated by our observations that waxcaps are less abundant in grassland with the ectomycorrhizal Helianthemum or Dryas (cf. Harrington 1996, 2003). Though the waxcaps in southern Canada and northern USA are predominantly found in ectomycorrhizal forests (Hesler \& Smith 1963: 35), it should be noted that temperate and boreal forests also hold endo-mycotrophic plants e.g. Taxus baccata or forbs such as Pulsatilla (Öpik et al. 2006). Moreover, ectomycorrhizal fungi in forests may be suppressed by shrubs colonised with arbuscular mycorrhizae (McHugh \& Gehring 2006). From Germany it is well known that some waxcaps are often associated with Fraxinus and Acer (Bresinsky et al. 2007, Krieglsteiner 2004), both being predominantly colonised by arbuscular fungi (Wang \& Qiu 2006). The dominant tree of the waxcap locations in Macaronesian laurel forests is Laurus novocanariensis, a species which is closely related to Laurus nobilis, also colonised by endophytic mycorrhizae (Maremmani et al. 2003). Unfortunately, waxcap habitat descriptions from the Americas only rarely consider the accompanying vegetation in sufficient detail. It seems that Hygrocybe avoids habitats where ectomycorrhizal fungi dominate. This could be interpreted as an avoidance of competition, which could imply a biotrophic mode on the side of the waxcaps as also suggested by Seitzman et al. (2011), corroborated by the reluctance of waxcaps to grow axenically (see introduction).

\section{Conclusion}

There are clear commonalities of the habitats investigated. "European" waxcaps prefer weakly developed soils of low fertility, temperate and boreal ecozones and grassland, without mosses being obligate associates. Still, some Hygrocybe species in our data set are widely distributed over almost every ecozone of our planet, occupying extremely diverse habitats. This and the peculier nutritional behaviour is in line with a biotrophic mode as suggested by Bresinsky (2008) and Seitzmann et al. (2011). Further research on the trophic lifestyle of waxcaps is needed, including histological investigations and barcoding of substrates and associated plants.

\section{Acknowledgements}

We thank Michael Beisenherz and Lothar Krieglsteiner for valuable information about waxcap ecology. Also Reinhard Agerer (Munich University), Claus Bässler (Bavarian Forest National Park) and Beatrice Senn-Irlet (Biodiversity and Conservation Biology, Swiss Federal Research Institute WSL) for insightful discussions about the waxcap enigma.

\section{References}

Andrews JH. 1992 - Fungal life-history strategies. In: The fungal community: its organization and role in the ecosystem (eds Carrol GC, Wicklow DT). Marcel Dekker, New York, 119145.

Arnolds E. 1981 - Ecology and coenology of macrofungi in grasslands and moist heathlands in Drenthe, the Netherlands. J. Cramer im AR Grantner Verlag.

Bailey RG. 1996 - Ecosystem geography. Springer, New York, NY (USA).

Beisenherz M. 2000 - Untersuchungen zur Ökologie und Systematik der Gattung Hygrocybe (Agaricales), doctoral thesis. Universität Regensburg.

BFN (Bundesamt für Naturschutz). 2004 - Interaktive CD-ROM zur Karte der natürlichen Vegetation Europas. http://www.floraweb.de/vegetation/dnld_eurovegmap.html

BMS. 2012 - The Fungal Records Database of Britain and Ireland. http://www.fieldmycology.net/FRDBI/FRDBI.asp. 
Boertmann D. 2010 - The Genus Hygrocybe. Svampetryk, Tilst.

Borgen T, Arnolds E. 2004 - Taxonomy, ecology and distribution of Hygrocybe (Fr.) P. Kumm. and Camarophyllopsis Herink (Fungi, Basidiomycota, Hygrocybeae) in Greenland. Monographs on Greenland - Meddelelser om Grønland. Bioscience 54, Copenhagen, the Danish Polar Center.

Bougher NL, Hart R, de Bueger S. 2006 - Whiteman Park Bushland, Fungi Report June 2006. Department of Environment and Conservation - Perth Urban Bushland Fungi Project.

Bresinsky A, Düring C, Ahlmer W. 2007 - PILZOEK 2. update. http://www.pilzoek.de.

Bresinsky A. 2008 - Die Gattungen Hydropus bis Hypsizygus mit Angaben zur Ökologie und Verbreitung der Arten. Regensburger mykologische Schriften.

Brunner I. 1987 - Pilzökologische Untersuchungen in Wiesen und Brachland in der Nordschweiz (Schaffhauser Jura). Veröffentlichungen des Geobotanischen Institutes der ETH, Stiftung Rübel, Zürich, 92. Heft.

Cardiff Council 2009 - Provisional atlas of waxcap fungi Hygrocybe in Cardiff. Strategic Planning and Environment Department. http://www.cardiff.gov.uk/objview.asp?object_id=14068.

CDMR (Consortium of Digital Mycology Resources). 2011 - Mushroom Observer.

Chanona-Gomez F, Andrade-Gallegos RH, Castellanos-Albores J, Sanchez JE. 2007 - Macrofungi from Parque Educativo Laguna Belgica, Municipality of Ocozocoautla de Espinosa, Chiapas, Mexico. Revista Mexicana de Biodiversidad 78(2), 369-381.

Cooke WB. 1979 - The ecology of fungi. CRC Press.

Dambrosio U. 2001 - Cocos Island moist forests, WWF Full Report. http://www.worldwildlife.org/wildworld/profiles/terrestrial/nt/nt0116_full.html.

Desjardin DE, Hemmes DE. 1997 - Agaricales of the Hawaiian Islands. 4: Hygrophoraceae. Mycologia 89(4), 615-638.

DFRA (Department of Forest Resources and Agrifoods). 2002 - Forest ecosystem strategy document for forest management district 1 (the Avalon peninsula), including Appendix 5 (Ecoregion Descriptions of Newfoundland \& Labrador). http://www.nr.gov.nl.ca/nr/forestry/forest/forest_types.html. http://www.nr.gov.nl.ca/nr/forestry/manage/appendix5.pdf http://www.nr.gov.nl.ca/nr/forestry/manage/sfm.pdf

EIC \& NCSE (Environmental Information Coalition \& The National Council for Science and the Environment). $2011-$ Encyclopedia of Earth. Boston University. http://www.eoearth.org/.

Eriksen M, Bjureke KE, Dhillion SS. 2002 - Mycorrhizal plants of traditionally managed boreal grasslands in Norway. Mycorrhiza 12(3), 117-123.

Escobar-Ocampo C, Ochoa-Gaona S. 2007 - Estructura y composición fl orística de la vegetación del Parque Educativo Laguna Bélgica, Chiapas, México. Revista Mexicana de Biodiversidad 78, 391-419.

FAO, ISRIC. 2009 - JRC Harmonized World Soil Database (version 1.1). FAO, Rome http://www.fao.org/nr/land/soils/harmonized-world-soil-database/en/

FAO. 2001 - Global ecological zoning for the global forest resources assessment 2000, final report. Forestry Department, Rome. ftp://ftp.fao.org/docrep/fao/006/ad652e/ad652e00.pdf

FAO. 2006 - Global forest resources assessment 2005. FAO Forestry Paper 147.

Favre J. 1960 - Catalogue descriptif des champignons supérieurs de la zone subalpine du Parc National Suisse. Résultats des rechcherches scientifiqes entreprises au Parc National Suisse 6, 323-610.

FPA. 2007 - Threatened Native Vegetation Community Information Sheet - cushion moorland. Forest Practice Authority, Hobart.

http://www.fpa.tas.gov.au/fileadmin/user_upload/PDFs/Botany/cushion_moorland_info _sheet_FE.pdf.

GBIF (Global Biodiversity Information Facility). 2011 - Data Portal. http://data.gbif.org/species/. 
Giri A, Rana P. 2007 - Some higher fungi from Sagarmatha National Park (SNP) and its adjoining areas, Nepal. Scientific World 5(5), 67-74.

Gómez LD. 1983 - The fungi of Cocos Island, Costa Rica. Brenesia 21, 355-364.

Greenpeace. 2006 - Roadmap to Recovery: The world's last intact forest landscapes. Greenpace International.

http://www.intactforests.org/pdf.publications/Greenpeace.World.IFL.2006.pdf

Griffith GW, Bratton JH, Easton G. 2004 - Charismatic megafungi - the conservation of waxcap grasslands. British Wildlife 16(1), 31-43.

Griffith GW, Easton GL, Jones AW. 2002 - Ecology and diversity of waxcap (Hygrocybe spp.) fungi. Transactions and Proceedings of the Botanical Society of Edinburgh and Botanical Society of Edinburgh Transactions 54(1), 7-22.

Griffith GW, Roderick K, Graham A, Causton DR. 2011 - Sward management influences fruiting of grassland basidiomycete fungi. Biological Conservation 145(1), 234-240.

Griffith GW, Roderick K. 2008 - Saprotrophic basidiomycetes in grasslands: distribution and function. In: Ecology of Saprotrophic Basidiomycetes (eds Boddy L, Frankland JC, van West P). Elsevier, 277-299.

Harrington T. 1996 - Observations on the macrofungi of Dryas communities in the Burren, western Ireland. The Irish Naturalists' Journal 25(5), 169-174.

Harrington T. 2003 - Relationships between macrofungi and vegetation in the Burren, The Royal Irish Academy 103B(3), 147-159.

Hesler LR, Smith AH. 1963 - North American species of Hygrophorus. The University of Tennessee Press, Knoxville.

Holden EM. 2007 - Fungal survey of Blairton Links. Aberdeenshire Menie Estate - Public Inquiry. Aberdeenshire Council. http://www.aberdeenshire.gov.uk/planning/inquiry/env_FungiReportPt2BlairtonLinks.p $\mathrm{df}$

Holden EM. 2007 - Fungal Survey of Blairton Links. Aberdeenshire Menie Estate - Public Inquiry, Aberdeenshire Council.

Homola RL, Czapowskyj MM, Blum BM. 1985 - Ectomycorrhizae of Maine: 3. A listing of Hygrophorus with the associated hosts (with additional information on edibility). Maine Agricultural Experiment Station Bulletin 810.

IUSS Working Group WRB. 2006 - World reference base for soil resources 2006. 2nd edition. World Soil Resources Reports No. 103. FAO, Rome. ftp://ftp.fao.org/agl/agll/docs/wsrr103e.pdf

Johnson CN. 2009 - Ecological consequences of Late Quaternary extinctions of megafauna. Proceedings of the Royal Society B: Biological Sciences 276(1667), 2509-2519.

Karasch P. 2001 - Beiträge zur Kenntnis der Pilzflora des Fünfseenlandes I. Ökologische Pilzkartierung auf einer Huteweide im Landkreis Weilheim. Zeitschrift für Mykologie 67(1), 73-136.

Karasch P. 2006 - Presentation at the International Meeting of the German Mycological Society (DGfM), Tübingen 2006.

Kearney R, Kearney E. 2007 - The listing of an Australian Hygrocybeae community: and its holotype species under State and Commonwealth legislations. Field Mycology 8(1), $13-21$.

Kehl H. 2011 - Vegetationsökologie Tropischer \& Subtropischer Klimate. TU-Berlin, Institut für Ökologie. twk.htm\#goFAOZones http://lv-twk.oekosys.tu-berlin.de/project/lv-twk/05-intro-4-

Kirby K. 2004 - A model of a natural wooded landscape in Britain as influenced by large herbivore activity. Forestry 77(5), 405-420.

Knudsen H, Vesterholt J. 2012 - Funga Nordica: Agaricoid, boletoid, clavaroid, cyphelloid and gastroid genera. Nordsvamp.

Köppen WP. 1931 - Grundriss der Klimakunde. W. de Gruyter Berlin. 
Kost G. 2002 - Contributions to tropical fungi I. Ecology and distribution of fungi of Kenya (East Africa). Feddes Repertorium 113(1-2), 132-151.

Krieglsteiner GJ, Gminder A. 2001 - Die Großpilze Baden-Württembergs. 3. Ständerpilze: Blätterpilze. Ulmer.

Krieglsteiner L. 2004 - Pilze im Biosphären-Reservat Rhön und ihre Einbindung in die Vegetation, doctoral thesis. Universität Regensburg.

Lepp H. 2001 - Mycologists and bryophytes. Australasian Mycologist 20(2), 76-78.

Lorenz M, Fischer R, Becher G, Mues V, Seidling W, Riedel T, Kraft P. 2006 - Forest condition in Europe. Technical Report of ICP Forests. Work Report Institute for World Forestry 2006/1.

Louro R, Calado M, Pinto B, Santos-Silva C. 2009 - Epigeous macrofungi of the Parque de Natureza de Noudar in Alentejo (Portugal). Mycotaxon 107, 49-52.

LRNZ (Landcare Research New Zealand). 2011 - NZFUNGI-New Zealand Fungi, Herbarium. http://nzfungi.landcareresearch.co.nz/html/search_collections.asp.

Maremmani A, Bedini S, Matoševic I, Tomei PE, Giovannetti M. 2003 - Type of mycorrhizal associations in two coastal nature reserves of the Mediterranean basin. Mycorrhiza 13(1), 33-40.

McGinley M. 2009 - Kahuzi-Biéga National Park, Democratic Republic of Congo. The Encyclopedia of $\quad$ Earth, http://www.eoearth.org/article/KahuziBi\%C3\%A9ga_National_Park,_Democratic_Republic_of_Congo.

McHugh R, Mitchel D, Wright M, Anderson R. 2001 - The fungi of Irish grasslands and their value for nature conservation. Biology and Environment: Proceedings of the Royal Irish Academy 101B/3, 225-243.

McHugh TA, Gehring CA. 2006 - Below-ground interactions with arbuscular mycorrhizal shrubs decrease the performance of pinyon pine and the abundance of its ectomycorrhizas. New Phytologist 171(1), 171-178.

McMullan-Fisher SJM, May TW, Kirkpatrick JB. 2003 - Some macrofungi from alpine Tasmania. Australasian Mycologist 22/1: 44-52.

Miller OK jr, Laursen GA, Farr DF. 1982 - Notes on Agaricales from Arctic tundra in Alaska. Mycologia 74(4), 576-591.

NBIC (Norwegian Biodiversity Information Centre). 2011 - Artsdatabanken. http://www.biodiversity.no/frontpage.aspx $? \mathrm{~m}=23$.

Öpik M, Moora M, Liira J, Zobel M. 2006 - Composition of root-colonizing arbuscular mycorrhizal fungal communities in different ecosystems around the globe. Journal of Ecology 94(4), 778-790.

Öster M. 2006 - Biological diversity values in semi-natural grasslands, doctoral thesis. University of Stockholm.

Öster M. 2008 - Low congruence between the diversity of waxcap (Hygrocybe spp.) fungi and vascular plants in semi-natural grasslands. Basic and Applied Ecology 9(5), 514-522.

Pugh G, Boddy L. 1988 - A view of disturbance and life strategies in fungi. Proceedings of the Royal Society of Edinburgh. Section B. Biological Sciences 94(1), 3-11.

Rotheroe M. 1999 - The Macrofungi of Dinefwr Park: A Pilot Study. Report to Countryside Council for Wales (CCW). http://www.aber.ac.uk/waxcap/downloads/rotheroe99dinefwr.pdf.

Salerni E, Perini C. 2007 - Mycodiversity of nature reserves in Central Italy. Acta Mycologica 42(1), 5-19.

Schultz J. 2008 - Die Ökozonen der Erde, 4. Auflage. Ulmer, Stuttgart.

Schweers A. 1949 - De Hygrophorusweide, een associatie. Fungus 19(2), 17-18.

Seitzman BH, Ouimette A, Mixon RL, Hobbie EA, Hibbett DS. 2011 - Conservation of biotrophy in Hygrophoraceae inferred from combined stable isotope and phylogenetic analyses. Mycologia 103(2), 280-290.

Selkirk PM, Adamson DA. 2011 - Map of structural vegetation types and drainage on subantarctic 
Macquarie Island. School of Biological Sciences, Macquarie University, N.S.W. 2109, Australia. http://data.aad.gov.au/metadata/other_data/macca_veg_map_doco.html.

Selkirk PM, Seppelt RD, Selkirk DR. 1990 - Subantarctic Macquarie Island: environment and biology. Cambridge University Press.

Sesli E. 2007 - Checklist of the Turkish ascomycota and basidiomycota collected from the Black Sea region. Mycotaxon 99, 71-74.

Simpson D, Butterbach-Bahl K, Fagerli H, Kesik M, Skiba U, Tang S. 2006 - Deposition and emissions of reactive nitrogen over European forests: a modelling study. Atmospheric Environment 40(29), 5712-5726.

Stabursvik EM. 2007 - The challenge of identifying and conserving valuable ecosystems close to human settlements in a northern area - An approach based on field- and satellite data. Master Thesis, Faculty of Science, Department of Biology, University of Troms $\varnothing$.

Svenning JC. 2002 - A review of natural vegetation openness in north-western Europe. Biological Conservation 104(2), 133-148.

TE ARA (NZ Ministry for Culture and Heritage). 2011 - Southern beech forest. http://www.teara.govt.nz/en/southern-beech-forest/1.

TOA (TEAM OF AUTHORS). 2011a - Mushroom Observer. http://mushroomobserver.org/name/observation_index.

TOA (TEAM OF AUTHORS). 2011b - Cybertruffle's Robigalia-Cybernome. http://www.cybertruffle.org.uk.

Tuininga AR. 2005 - Interspecific interaction terminology: from mycology to general ecology. In Dighton J, White Jr J, Oudemans P. The fungal community: its organization and role in the ecosystem, Third Edition. - CRC Press: 265-283.

Vera FWM. 2000 - Grazing ecology and forest history. CABI.

Voitk A. 2005 - Mycological Avalon - report of a brief, superficfial \& partial survey of four provincial parks for suitable use in a mushroom foray. http://www.hnhs.ca/wpcontent/UserFiles/File/foray2006/AvalonReport.pdf.

Walter H, Breckle SW. 1999 - Vegetation und Klimazonen. Ulmer, Stuttgart.

Wang B, Qiu YL. 2006 - Phylogenetic distribution and evolution of mycorrhizas in land plants. Mycorrhiza 16(5), 299-363.

WP (WHITEMAN $\quad$ PARK). $2011 \quad-\quad$ Conservation Reserve. http://www.whitemanpark.com.au/conservationreserve.aspx.

Young T. 2005 - Fungi of Australia: Hygrophoraceae. CSIRO. 